\title{
Cloaking Devices, Electromagnetic Wormholes and Transformation Optics
}

\author{
Allan Greenleaf* \\ Yaroslav Kurylev ${ }^{\dagger}$ \\ Matti Lassas, ${ }^{\ddagger}$ \\ Gunther Uhlmann ${ }^{\S}$
}

\begin{abstract}
We describe recent theoretical and experimental progress on making objects invisible to detection by electromagnetic waves. Ideas for devices that would have once seemed fanciful may now be at least approximately implemented physically using a new class of artificially structured materials called metamaterials. Maxwell's equations have transformation laws that allow for design of electromagnetic material parameters that steer light around a hidden region, returning it to its original path on the far side. Not only would observers be unaware of the contents of the hidden region, they would not even be aware that something was being hidden. The object, which would have no shadow, is said to be cloaked. Proposals for, and even experimental implementations of, such cloaking devices have received the most attention, but other designs having striking effects on wave propagation are possible. All of these designs are initially based on the transformation laws of the equations that govern wave propagation but, due
\end{abstract}

*Department of Mathematics, University of Rochester, Rochester, NY 14627, USA. Partially supported by NSF grant DMS-0551894.

${ }^{\dagger}$ Department of Mathematics, University College London, Gower Street, London, WC1E 5BT, UK. Partially supported by EPSRC grant EP/F0340116.

${ }^{\ddagger}$ Helsinki University of Technology, Institute of Mathematics, P.O.Box 1100, FIN02015, Finland. Partially supported by Academy of Finland CoE Project 213476.

$\S$ Department of Mathematics, University of Washington, Seattle, WA 98195, USA. Partially supported by the NSF and a Walker Family Endowed Professorship. 
to the singular parameters that give rise to the desired effects, care needs to be taken in formulating and analyzing physically meaningful solutions. We recount the recent history of the subject and discuss some of the mathematical and physical issues involved.

\section{Introduction}

Invisibility has been a subject of human fascination for millenia, from the Greek legend of Perseus versus Medusa to the more recent The Invisible Man and Harry Potter. Over the years, there have been occasional scientific prescriptions for invisibility in various settings, e.g., [46, 7]. However, since 2005 there has been a wave of serious theoretical proposals $[1,72,69,65,80]$ in the physics literature, and a widely reported experiment by Schurig et al. [88], for cloaking devices - structures that would not only render an object invisible but also undetectable to electromagnetic waves. The particular route to cloaking that has received the most attention is that of transformation optics [101], the designing of optical devices with customized effects on wave propagation, made possible by taking advantage of the transformation rules for the material properties of optics: the index of refraction $n(x)$ for scalar optics, governed by the Helmholtz equation, and the electric permittivity $\varepsilon(x)$ and magnetic permeability $\mu(x)$ for vector optics, as described by Maxwell's equations. It is this approach to cloaking that we will examine in some detail.

As it happens, two papers appeared in the same issue of Science with transformation optics-based proposals for cloaking. Leonhardt [65] gave a description, based on conformal mapping, of inhomogeneous indices of refraction $n$ in two dimensions that would cause light rays to go around a region and emerge on the other side as if they had passed through empty space (for which $n \equiv 1$ ). (The region in question is then said to be cloaked.) On the other hand, Pendry, Schurig and Smith [80] gave a prescription for values of $\varepsilon$ and $\mu$ giving a cloaking device for electromagnetic waves, based on the fact that $\varepsilon$ and $\mu$ transform in the same way (7) as the conductivity tensor in electrostatics. In fact, they used exactly the same singular transformation (15), resulting in singular electromagnetic material parameters, as had already been used three years earlier to describe examples of nondetectability in the context of the Calderón Problem [38, 39]! 
Science magazine stated, in its ranking of cloaking as the No. 5 Breakthrough of 2006 ("The Ultimate Camouflage"),

"... The real breakthrough may lie in the theoretical tools used to make the cloak. In such "transformation optics," researchers imagine - á la Einstein - warping empty space to bend the path of electromagnetic waves. A mathematical transformation then tells them how to mimic the bending by filling unwarped space with a material whose optical properties vary from point to point. The technique could be used to design antennas, shields, and myriad other devices. Any way you look at it, the ideas behind invisibility are likely to cast a long shadow."

The papers $[38,39]$ considered the case of electrostatics, which can be considered as optics at frequency zero. In $\S 2$ we describe this case in more detail since it already contains the basic idea of transformation optics and also shows the importance of careful formulation and analysis of solutions in the setting of singular transformation optics. These articles give counterexamples to uniqueness in Calderón's Problem, which is the inverse problem for electrostatics which lies at the heart of Electrical Impedance Tomography. This consists in determining the electrical conductivity of a medium filling a region $\Omega$ by making voltage and current measurements at the boundary $\partial \Omega$. The counterexamples were motivated by consideration of certain degenerating families of Riemannian metrics, which in the limit correspond to singular conductivities, i.e., that are not bounded below or above, so that the corresponding PDE is no longer uniformly elliptic. A related example of a complete but noncompact two-dimensional Riemannian manifold with boundary having the same Dirichlet-Neumann map as a compact one was given in [62]. The techniques in $[38,39]$ are valid in dimensions three and higher, but the same construction has been shown to work in two dimensions [55]. We point out here that although we emphasize boundary observations using the Dirichlet-Neumann map or the set of Cauchy data, this is equivalent to scattering information [6]; see [98].

In considering wave propagation, one can either work in the frequency domain or the time domain. Because the metamaterials that have been proposed for use in cloaking (and more general transformation optics designs) are inherently prone to dispersion, i.e., their material parameters $n, \varepsilon$ and 
$\mu$ are frequency-dependent, and only have the desired values over relatively narrow bandwidths, it is natural to work in the frequency domain, with time-harmonic waves of frequency $k$. Further comments on the time-domain approach are in $\S 7(\mathrm{~d})$.

In $\S 3$ we consider cloaking for the Helmholtz equation and Maxwell's equations. We place special emphasis on the behavior of the waves near the boundary of the cloaked region. This is crucial given that the electromagnetic parameters are singular at this cloaking surface. The analysis of $[65,81]$ uses ray tracing which explains the behavior of the light rays but not the full electromagnetic waves. The article [80] analyses the behavior of the waves outside the cloaked region, using the transformation law for solutions to Maxwell's equations under smooth transformations, which unfortunately is not valid at the cloaking surface. The article [26], which gave numerical simulations of the electromagnetic waves in the presence of a cloak, states: "Whether perfect cloaking is achievable, even in theory, is also an open question". In [32], perfect cloaking was shown to indeed hold with respect to finite energy distribution solutions of Maxwell's equations, with passive objects (no internal currents) being cloaked (see Theorem 3.4 below). The electromagnetic material parameters used are the push-forward of a homogeneous, isotropic medium by a singular transformation that "blows up" a point to the cloaking surface. This is referred to in [32] as the single coating construction and is the same "spherical cloak" as described in [38, 39, 80]. We also analyze the case of cloaking active objects for both Helmholtz's equation and Maxwell's equations. For Helmholtz, such cloaking is always possible ${ }^{1}$, but for Maxwell certain overdetermined boundary conditions emerge at the cloaking surface. While satisfied for passive cloaked objects, they cannot be satisfied for generic internal currents, i.e., for active objects that are themselves radiating within the cloaked region. However, the situation can be rectified by either installing a lining at the cloaking surface, or by using a double coating, which corresponds to matched metamaterials on both sides of the cloaking surface, while the construction above is what we call the single coating [32]. This theoretical description of an invisibility device can, in principle, be physically realized by taking an arbitrary object in $N_{2}$ and surrounding it with special material, located in $N_{1}$, which implements the values of $\widetilde{\varepsilon}, \widetilde{\mu}$. The materials

\footnotetext{
${ }^{1}$ Since Helmholtz also governs acoustic waves, this allows the theoretical description of a 3D acoustic cloak, a spherically symmetric case of which was subsequently obtained in the physics literature [22, 28]; see [36].
} 
proposed for cloaking with electromagnetic waves are artificial materials referred to as metamaterials. The study of these material has undergone an explosive growth in recent years. There is no universally accepted definition of metamaterials, which seem to be in the "know it when you see it" category. However, the label usually attaches to macroscopic material structures having a manmade one-, two- or three-dimensional cellular architecture, and producing combinations of material parameters not available in nature (or even in conventional composite materials), due to resonances induced by the geometry of the cells $[100,30]$. Using metamaterial cells (or "atoms", as they are sometimes called), designed to resonate at the desired frequency, it is possible to specify the permittivity and permeability tensors fairly arbitrarily at a given frequency, so that they may have very large, very small or even negative eigenvalues, cf. $\S 7(\mathrm{i})$. The use of resonance phenomenon also explains why the material properties of metamaterials strongly depend on the frequency, and broadband metamaterials may not be possible.

In $\S 4$ we consider the case of cloaking an infinite cylinder for Maxwell's equations; the experiment [88] was designed to implement a "reduced" set of material parameters, easier to construct but replicating a $2 \mathrm{D}$ slice of the ray geometry of the mathematical ideal. To ensure that the solutions of Maxwell's equations are well defined in the case of the cylindrical cloaking, we will consider the single coating construction with a lining to enforce the Soft-and-Hard Boundary (SSH) boundary conditions considered by Kildal $[47,48]$, see also [67]. If these conditions are not satisfied the fields blow up $[87,34]$, and this has important implications for approximate cloaking, the analysis of the behavior of waves in the presence of less-than-perfect cloaks. We should point out that serious skepticism concerning the practical advantages of transformation optics based cloaking over earlier techniques for reducing scattering has been expressed in the engineering community [49]. Exactly how effective cloaking and transformation optics devices will be in practice is very much at the mercy of future improvements in the design, analysis and fabrication of metamaterials.

In $\S 5$ we describe the electromagnetic wormholes introduced in $[33,35]$ which allow for an invisible tunnel between two points in space. Electromagnetic waves are tricked by the metamaterial specification into behaving as though they were propagating on a handlebody, rather than on $\mathbb{R}^{3}$. The prescription of appropriate metamaterials covering and filling a cylinder and producing this behavior is obtained using a pair of singular transformations that effec- 
tively blow up a curve rather than a point. For popular accounts of this work see $[83,43,97]$.

In $\S 6$, we describe a framework for a less ad hoc approach to transformation optics when the transformation fails to be smooth and the chain rule no longer fully applies; we refer to this as singular transformation optics. Ultimately, the fundamental justification for a singular transformation optics-based device will be, just as for cloaking and the wormhole, a removable singularities theorem. Finally, in $\S 7$, we discuss some of the other recent progress in cloaking and transformation optics.

\section{The case of electrostatics: Calderón's problem}

Calderón's inverse problem, which forms the mathematical foundation of Electrical Impedance Tomography (EIT), is the question of whether an unknown conductivity distribution inside a domain in $\mathbb{R}^{n}$, modelling for example the Earth, a human thorax, or a manufactured part, can be determined from voltage and current measurements made on the boundary. A.P. Calderón's motivation to propose this problem [19] was geophysical prospection. In the 1940's, before his distinguished career as a mathematician, Calderón was an engineer working for the Argentinian state oil company "Yacimientos Petrolíferos Fiscales" (YPF). Apparently, at that time Calderón had already formulated the problem that now bears his name, but did not publicize his work until thirty years later.

One widely studied potential application of EIT is the early diagnosis of breast cancer [24]. The conductivity of a malignant breast tumor is typically 0.2 mho, significantly higher than normal tissue, which has been typically measured at 0.03 mho. See the book [41] and the special issue of Physiological Measurement [42] for applications of EIT to medical imaging and other fields.

For isotropic conductivities this problem can be mathematically formulated as follows: Let $\Omega$ be the measurement domain, and denote by $\sigma(x)$ the coefficient, bounded from above and below by positive constants, describing the electrical conductivity in $\Omega$. In $\Omega$ the voltage potential $u$ satisfies a divergence form equation,

$$
\nabla \cdot \sigma \nabla u=0
$$



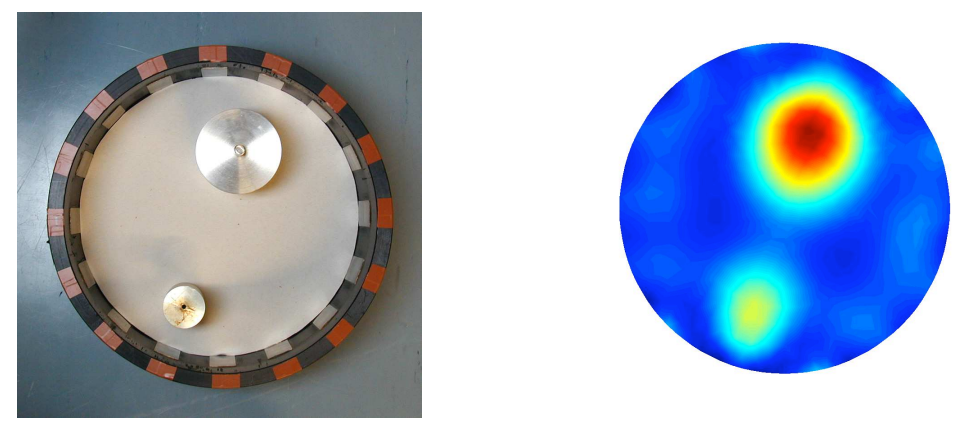

Figure 1: Left: An EIT measurement configuration for imaging objects in a tank. The electrodes used for measurements are at the boundary of the tank, which is filled with a conductive liquid. Right: A reconstruction of the conductivity inside the tank obtained using boundary measurements. [Jari Kaipio, Univ. of Kuopio, Finland; by permission.]

To uniquely fix the solution $u$ it is enough to give its value, $f$, on the boundary. In the idealized case, one measures, for all voltage distributions $\left.u\right|_{\partial \Omega}=f$ on the boundary the corresponding current fluxes, $\nu \cdot \sigma \nabla u$, over the entire boundary, where $\nu$ is the exterior unit normal to $\partial \Omega$. Mathematically this amounts to the knowledge of the Dirichlet-Neumann (DN) map, $\Lambda_{\sigma}$. corresponding to $\sigma$, i.e., the map taking the Dirichlet boundary values of the solution to (1) to the corresponding Neumann boundary values,

$$
\Lambda_{\sigma}:\left.\left.\quad u\right|_{\partial \Omega} \mapsto \nu \cdot \sigma \nabla u\right|_{\partial \Omega} .
$$

Calderón's inverse problem is then to reconstruct $\sigma$ from $\Lambda_{\sigma}$.

\subsection{Conductivities that do not cloak}

For what conductivities is there no cloaking? This is the question of uniqueness of determination of the conductivity from the DN map. We first consider the isotropic case. Kohn and Vogelius showed that piecewise analytic conductivities are uniquely determine by the DN map [57]. Sylvester and Uhlmann proved that $C^{\infty}$ smooth conductivities can be uniquely determined by the DN map in dimension $n \geq 3$. This was extended to conductivities having $3 / 2$ 
derivatives [79, 14], which is the best currently known result for scalar conductivities for $n \geq 3$. For conormal conductivities in $C^{1+\epsilon}$, uniqueness was shown in [37]. In the challenging two dimensional case, unique identifiability of the conductivity from the DN map was shown for $C^{2}$ conductivities by Nachman [74], for Lipschitz conductivities by Brown and Uhlmann [15], and for the optimal class of merely $L^{\infty}$ conductivities by Astala and Päivärinta [2]. We are only briefly summarizing here the known uniqueness results for isotropic conductivities since, as will be seen below, these are not directly relevant to the subject of cloaking. For issues concerning stability, analytic and numerical reconstruction in EIT see the surveys [8], [24], [99].

We now discuss the anisotropic case, that is when the conductivity depends on direction. Physically realistic models must incorporate anisotropy. In the human body, for example, muscle tissue is a highly anisotropic conductor, e.g., cardiac muscle has a conductivity of 2.3 mho in the direction transversal to the fibers and 6.3 mho in the longitudinal direction.

An anisotropic conductivity on a domain $\Omega \subset \mathbb{R}^{n}$ is defined by a symmetric, positive semi-definite matrix-valued function, $\sigma=\left[\sigma^{i j}(x)\right]_{i, j=1}^{n}$. In the absence of sources or sinks, an electrical potential $u$ satisfies

$$
\begin{aligned}
(\nabla \cdot \sigma \nabla) u=\partial_{j} \sigma^{j k}(x) \partial_{k} u & =0 \text { in } \Omega, \\
\left.u\right|_{\partial \Omega} & =f,
\end{aligned}
$$

where $f$ is the prescribed voltage on the boundary. (Above, and hereafter, we use the Einstein summation convention when there is no danger of confusion.) The resulting DN map (or voltage-to-current map) is then defined by

$$
\Lambda_{\sigma}(f)=\left.B u\right|_{\partial \Omega}
$$

where

$$
B u=\nu_{j} \sigma^{j k} \partial_{k} u
$$

$u$ being the solution of $(3)$ and $\nu=\left(\nu_{1}, \ldots, \nu_{n}\right)$ the unit normal vector of $\partial \Omega$. Applying the divergence theorem, we have

$$
Q_{\sigma}(f)=: \int_{\Omega} \sigma^{j k}(x) \frac{\partial u}{\partial x^{j}} \frac{\partial u}{\partial x^{k}} d x=\int_{\partial \Omega} \Lambda_{\sigma}(f) f d S,
$$

where $u$ solves (3) and $d S$ denotes surface measure on $\partial \Omega . Q_{\sigma}(f)$ represents the power needed to maintain the potential $f$ on $\partial \Omega$. By (6), knowing $Q_{\sigma}$ 
is equivalent with knowing $\Lambda_{\sigma}$. If $F: \Omega \rightarrow \Omega, F=\left(F^{1}, \ldots, F^{n}\right)$, is a diffeomorphism with $\left.F\right|_{\partial \Omega}=$ Identity, then by making the change of variables $y=F(x)$ and setting $u=v \circ F^{-1}$ in the first integral in (6), we obtain

$$
\Lambda_{F_{*} \sigma}=\Lambda_{\sigma}
$$

where

$$
\left(F_{*} \sigma\right)^{j k}(y)=\left.\frac{1}{\operatorname{det}\left[\frac{\partial F^{j}}{\partial x^{k}}(x)\right]} \sum_{p, q=1}^{n} \frac{\partial F^{j}}{\partial x^{p}}(x) \frac{\partial F^{k}}{\partial x^{q}}(x) \sigma^{p q}(x)\right|_{x=F^{-1}(y)}
$$

is the push-forward of the conductivity $\sigma$ by $F$. Thus, there is a large (infinite-dimensional) class of conductivities which give rise to the same electrical measurements at the boundary. This was first observed in [58] following a remark by Luc Tartar. The version of Calderón's problem appropriate for anisotropic conductivities is then the question of whether two conductivities with the same DN map must be such push-forwards of each other.

It was observed by Lee and Uhlmann [64] that, in dimension $n \geq 3$, the anisotropic problem can be reformulated in geometric terms. Let us assume now that $(M, g)$ is an $n$-dimensional Riemannian manifold with smooth boundary $\partial M$. The metric $g$ is assumed to be symmetric and positive definite. The invariant object analogous to the operator in conductivity equation (3) is the Laplace-Beltrami operator, given by

$$
\Delta_{g} u=\operatorname{Div}_{g} \operatorname{Grad}_{g} u=|g|^{-1 / 2} \partial_{j}\left(|g|^{1 / 2} g^{j k} \partial_{k} u\right)
$$

where $|g|=\operatorname{det}\left(g_{j k}\right),\left[g_{j k}\right]=\left[g^{j k}\right]^{-1}$. The DN map is defined by solving the Dirichlet problem

$$
\Delta_{g} u=0 \quad \text { in } \quad M,\left.\quad u\right|_{\partial M}=f .
$$

The operator analogous to $\Lambda_{\sigma}$ is then

$$
\Lambda_{g}(f)=\left.|g|^{1 / 2} \nu_{j} g^{j k} \frac{\partial u}{\partial x_{k}}\right|_{\partial M},
$$

with $\nu=\left(\nu_{1}, \ldots, \nu_{n}\right)$ the outward unit normal to $\partial M$. In dimension three or higher, the conductivity matrix and the Riemannian metric are related by

$$
\sigma^{j k}=|g|^{1 / 2} g^{j k}, \quad \text { or } \quad g^{j k}=\operatorname{det}(\sigma)^{2 /(n-2)} \sigma^{j k} .
$$


Moreover,

$$
\Lambda_{g}=\Lambda_{\sigma} ; \quad \Lambda_{F_{*} g}=\Lambda_{g}
$$

where $F_{*} g$ denotes the push-forward of the metric $g$ by a diffeomorphism $F$ of $M$ fixing $\partial M$ [64]. We recall that in local coordinates

$$
\left(F_{*} g\right)_{j k}(y)=\left.\sum_{p, q=1}^{n} \frac{\partial F^{p}}{\partial x^{j}}(x) \frac{\partial F^{q}}{\partial x^{k}}(x) g_{p q}(x)\right|_{x=F^{-1}(y)} .
$$

In dimension two, (12) is not valid; in this case, the conductivity equation can be reformulated as

$$
\begin{aligned}
& \operatorname{Div}_{g}\left(\beta \operatorname{Grad}_{g} u\right)=0 \quad \text { in } \quad M, \\
& \left.u\right|_{\partial M}=f
\end{aligned}
$$

where $\beta$ is the scalar function $\beta=|\operatorname{det} \sigma|^{1 / 2}, g=\left(g_{j k}\right)$ is equal to $\left(\sigma_{j k}\right)$, and $\operatorname{Div}_{g}$ and $\operatorname{Grad}_{g}$ are the divergence and gradient operators with respect to the Riemannian metric $g$. Thus we see that, in two dimensions, LaplaceBeltrami operators correspond only to those conductivity equations for which $\operatorname{det}(\sigma)=1$.

For domains in two dimensions, Sylvester [95] showed, using isothermal coordinates, that one can reduce the anisotropic problem to the isotropic one for $C^{3}$ conductivities. This reduction was extended to Lipschitz conductivities in [94] using the result of [15] and to bounded conductivities in [3], using the result of [2]. The result of [3] is

Theorem 2.1 If $\sigma$ and $\widetilde{\sigma}$ are two $L^{\infty}$ anisotropic conductivities bounded from below by a positive constant in a bounded set $\Omega \subset \mathbb{R}^{2}$ for which $\Lambda_{\sigma}=\Lambda_{\tilde{\sigma}}$, then there is a diffeomorphism $F: \Omega \rightarrow \Omega,\left.F\right|_{\partial \Omega}=I d$ such that $\widetilde{\sigma}=F_{*} \sigma$.

In dimensions three and higher, the uniqueness result is known for real analytic anisotropic conductivities or metrics (see [61], [62], and [64]):

Theorem 2.2 If $n \geq 3$ and $(M, \partial M)$ is a $C^{\omega}$ manifold with a non-empty, compact, $C^{\omega}$ boundary, and $g, \widetilde{g}$ are $C^{\omega}$ metrics on $M$ such that $\Lambda_{g}=\Lambda_{\widetilde{g}}$, then there exists a $C^{\omega}$ diffeomorphism $F: M \rightarrow M$ such that $\left.F\right|_{\partial D}=I d$ and $\widetilde{g}=F_{*} g$. 
We also mention that the invariance of the Dirichlet-Neumann map under changes of variables was used in [53] to find the unique isotropic conductivity that is closest to an anisotropic one.

A problem related to Calderón's problem is the Gel'fand problem, which uses boundary measurements at all frequencies, rather than at a fixed one. For this problem uniqueness results are available; see, e.g., $[5,44]$, with a detailed exposition in [45].

\subsection{Transformation Optics for Electrostatics}

The fact that smooth diffeomorphisms that leave the boundary fixed give the same boundary information (12) can already be considered as a weak form of invisibility, with distinct conductivities being indistinguishable by external observations; however, nothing has been hidden yet.

Using the invariance (12) examples of singular anisotropic conductivities in $\mathbb{R}^{n}, n \geq 3$, that are indistinguishable from a constant isotropic conductivity, in that they have the same Dirichlet-to-Neumann map, are given in [38, 39]. This construction is based on degenerations of Riemannian metrics, whose singular limits can be considered as coming from singular changes of variables.

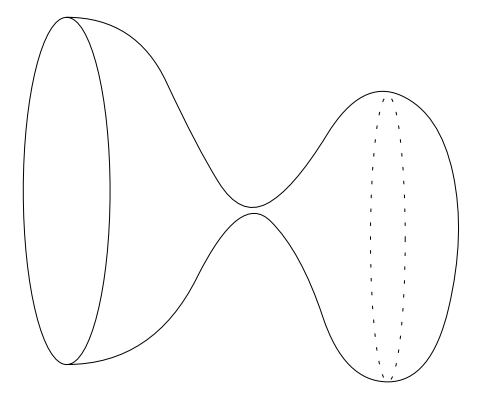

Figure 2: A typical member of a family of manifolds developing a singularity as the width of the neck connecting the two parts goes to zero.

If one considers Fig. 2, where the "neck" of the surface (or a manifold in the higher dimensional cases) is pinched, the manifold contains in the limit a pocket about which the boundary measurements do not give any information. 
If the collapsing of the manifold is done in an appropriate way, in the limit we have a (singular) Riemannian manifold which is indistinguishable from a flat surface. This can be considered as a conductivity, singular at the pinched points, that appears to all boundary measurements the same as a constant conductivity.

To give a precise realization of this idea, let $B(0, R) \subset \mathbb{R}^{3}$ be an open ball with center 0 and radius $R$. We use in the sequel the set $N=B(0,2)$, decomposed to two parts, $N_{1}=B(0,2) \backslash \bar{B}(0,1)$ and $N_{2}=B(0,1)$. Let $\Sigma=\partial N_{2}$ the the interface (or "cloaking surface") between $N_{1}$ and $N_{2}$.

We use also a "copy" of the ball $B(0,2)$, with the notation $M_{1}=B(0,2)$. Let $g_{j k}=\delta_{j k}$ be the Euclidian metric in $M_{1}$ and let $\gamma=1$ be the corresponding homogeneous conductivity. Define a singular transformation

$$
F_{1}: M_{1} \backslash\{0\} \rightarrow N_{1}, \quad F_{1}(x)=\left(\frac{|x|}{2}+1\right) \frac{x}{|x|}, \quad 0<|x| \leq 2 .
$$

The push-forward $\widetilde{g}=\left(F_{1}\right)_{*} g$ of the metric $g$ by $F_{1}$ is the metric in $N_{1}$ given by

$$
\left(\left(F_{1}\right)_{*} g\right)_{j k}(y)=\left.\sum_{p, q=1}^{n} \frac{\partial F_{1}^{p}}{\partial x^{j}}(x) \frac{\partial F_{1}^{q}}{\partial x^{k}}(x) g_{p q}(x)\right|_{x=F_{1}^{-1}(y)}
$$

We use it to define a singular conductivity

$$
\widetilde{\sigma}= \begin{cases}|\widetilde{g}|^{1 / 2} \widetilde{g}^{j k} & \text { for } x \in N_{1} \\ \delta^{j k} & \text { for } x \in N_{2}\end{cases}
$$

in $N$. (The way to think of $\widetilde{\sigma}$ on $N_{2}$ is that it is the pushforward of $\delta^{j k}$ under the identity map $F_{2}: M_{2} \stackrel{\text { def }}{=} B(0,1) \longrightarrow N_{2}$, which could in fact be replaced by any diffeomorphism "filling in the hole" left by $F_{1}$.)

To consider the maps $F_{1}$ and $F_{2}$ together, let $M$ be the disjoint union of a ball $M_{1}=B(0,2)$ and a ball $M_{2}=B(0,1)$. These will correspond to sets $N, N_{1}, N_{2}$ after an appropriate changes of coordinates. We thus consider a map $F: M \backslash\{0\}=\left(M_{1} \backslash\{0\}\right) \cup M_{2} \rightarrow N \backslash \Sigma$, where $F$ maps $M_{1} \backslash\{0\}$ to $N_{1}$ as the map $F_{1}$ defined by formula (15) and $F$ maps from $M_{2}$ to $N_{2}$ as the identity map $F_{2}=I d$. The combined map, $F=\left(F_{1}, F_{2}\right)$, "blows up a point". Using spherical coordinates, $(r, \phi, \theta) \mapsto(r \sin \theta \cos \phi, r \sin \theta \sin \phi, r \cos \theta)$, we 
have

$$
\widetilde{\sigma}=\left(\begin{array}{ccc}
2(r-1)^{2} \sin \theta & 0 & 0 \\
0 & 2 \sin \theta & 0 \\
0 & 0 & 2(\sin \theta)^{-1}
\end{array}\right), \quad 1<|x| \leq 2
$$

This means that in the Cartesian coordinates the conductivity $\widetilde{\sigma}$ is given by

$$
\widetilde{\sigma}(x)=2(I-P(x))+2|x|^{-2}(|x|-1)^{2} P(x), \quad 1<|x|<2,
$$

where $I$ is the identity matrix and $P(x)=|x|^{-2} x x^{t}$ is the projection to the radial direction. We note that the anisotropic conductivity $\widetilde{\sigma}$ is singular on $\Sigma$ in the sense that it is not bounded from below by any positive multiple of I. (See [55] for a similar calculation.)

Consider now the Cauchy data of all solutions in the Sobolev space $H^{1}(N)$ of the conductivity equation corresponding to $\widetilde{\sigma}$, that is,

$$
C_{1}(\widetilde{\sigma})=\left\{\left(\left.u\right|_{\partial N},\left.\nu \cdot \widetilde{\sigma} \nabla u\right|_{\partial N}\right): u \in H^{1}(N), \nabla \cdot \tilde{\sigma} \nabla u=0\right\},
$$

where $\nu$ is the Euclidian unit normal vector of $\partial N$.

Theorem 2.3 ([39]) The Cauchy data of all $\mathrm{H}^{1}$-solutions for the conductivities $\widetilde{\sigma}$ and $\gamma$ on $N$ coincide, that is, $C_{1}(\widetilde{\sigma})=C_{1}(\gamma)$.

This means that all boundary measurements for the homogeneous conductivity $\gamma=1$ and the degenerated conductivity $\widetilde{\sigma}$ are the same. The result above was proven in $[37,38]$ for the case of dimension $n \geq 3$. The same basic construction works in the two dimensional case [55]. For a further study of the limits of visibility and invisibility in two dimensions, see [4].

Fig. 3 portrays an analytically obtained solution on a disc with conductivity $\widetilde{\sigma}$. As seen in the figure, no currents appear near the center of the disc, so that if the conductivity is changed near the center, the measurements on the boundary $\partial N$ do not change.

Remark 2.4 We now make a simple but crucial observation: In order for the one-to-one correspondence between solutions of the conductivity equation for $\gamma$ and those for $\widetilde{\sigma}$ to hold, it is necessary to impose some regularity assumption on the electrical potentials $\widetilde{u}$ for $\widetilde{\sigma}$. If, for example, we start with the Newtonian potential $K(x)=-\frac{1}{4 \pi|x|}$, then this pushes forward to a 


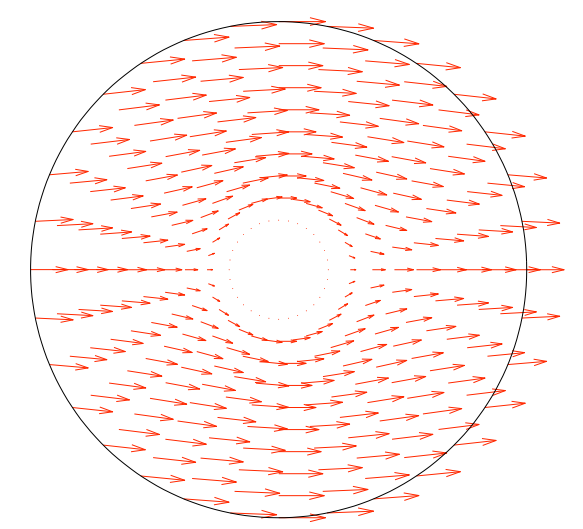

Figure 3: Analytic solutions for the currents

(non- $H^{1}$ ) potential for $\widetilde{\sigma}$ whose Cauchy data do not equal the Cauchy data of any potential $u$ for $\gamma$. Thus, it does not suffice to simply appeal to the transformation law (7) in the exterior of the cloaked region. This comment is equally valid when one considers cloaking for the Helmholtz and Maxwell's equations.

The invisibility result is valid for a more general class of singular cloaking transformations. Quadratic singular transformations for Maxwell's equations were introduced first in [18]. A general class sufficing, at least for electrostatics, is given by the following result from [38]:

Theorem 2.5 Let $\Omega \subset \mathbb{R}^{n}, n \geq 3$ be a bounded domain with a smooth boundary, $y \in \Omega$, and $g=\left(g_{i j}\right)$ a metric on $\Omega$. Let $D \subset \Omega$ be such that there is a $C^{\infty}$-diffeomorphism $F: \Omega \backslash\{y\} \rightarrow \Omega \backslash \bar{D}$ satisfying $\left.F\right|_{\partial \Omega}=$ Id and that

$$
d F(x) \geq c_{0} I, \quad \operatorname{det}(d F(x)) \geq c_{1} \operatorname{dist}_{\mathbb{R}^{n}}(x, y)^{-1}
$$

where $d F$ is the Jacobian matrix in Euclidean coordinates of $\mathbb{R}^{n}$ and $c_{0}, c_{1}>$ 0 . Let $\widetilde{g}=F_{*} g$ and $\widehat{g}$ be an extension of $\widetilde{g}$ into $D$ such that it is positive definite in $D^{\text {int }}$. Finally, let $\gamma$ and $\widehat{\sigma}$ be the conductivities corresponding to $g$ and $\widehat{g}$. Then,

$$
C_{1}(\widehat{\sigma})=C_{1}(\gamma)
$$


The key to the proof of Theorem 2.5 is the following removable singularities theorem that implies that solutions of the conductivity equation in the annulus pull back by a singular transformation to solutions of the conductivity equation in the whole ball.

Proposition 2.6 Let $\Omega \subset \mathbb{R}^{n}, n \geq 3$ be a bounded domain with a smooth boundary, $y \in \Omega$, and $g=g_{i j}$ a metric on $\Omega$. Let $u$ satisfy

$$
\begin{aligned}
& \Delta_{g} u(x)=0 \quad \text { in } \Omega, \\
& \left.u\right|_{\partial \Omega}=f_{0} \in C^{\infty}(\partial \Omega) .
\end{aligned}
$$

Let $D \subset \Omega$ be such that there is a diffeomorphism $F: \Omega \backslash\{y\} \rightarrow \Omega \backslash \bar{D}$ satisfying $\left.F\right|_{\partial \Omega}=I d$. Let $\widetilde{g}=F_{*} g$ and $v$ be a function satisfying

$$
\begin{aligned}
& \Delta_{\tilde{g}} v(x)=0 \quad \text { in } \Omega \backslash \bar{D}, \\
& \left.v\right|_{\partial \Omega}=f_{0}, \\
& v \in L^{\infty}(\Omega \backslash \bar{D}) .
\end{aligned}
$$

Then $u$ and $F^{*} v$ coincide and have the same Cauchy data on $\partial \Omega$,

$$
\left.\partial_{\nu} u\right|_{\partial M}=\left.\partial_{\widetilde{\nu}} F^{*} v\right|_{\partial M}
$$

where $\nu$ is unit normal vector in metric $g$ and $\widetilde{\nu}$ is unit normal vector in metric $\widetilde{g}$.

Quadratic singular transformations, such as

$$
F(x)=\left(1+|x|^{2}\right) \frac{x}{|x|}
$$

were used in [18] to reduce exterior reflections. We note that a similar type of theorem is valid also for a more general class of solutions. Consider an unbounded quadratic form, $A$ in $L^{2}(N)$,

$$
A_{\widetilde{\sigma}}[u, v]=\int_{N} \tilde{\sigma} \nabla u \cdot \nabla v d x
$$

defined for $u, v \in \mathcal{D}\left(A_{\widetilde{\sigma}}\right)=C_{0}^{\infty}(N)$. Let $\bar{A}_{\widetilde{\sigma}}$ be the closure of this quadratic form and say that 


$$
\begin{aligned}
\nabla \cdot \tilde{\sigma} \nabla u & =0 \text { in } N \\
\left.u\right|_{\partial N} & =f_{0}
\end{aligned}
$$

is satisfied in the finite energy sense if there is $u_{0} \in H^{1}(N)$ supported in $N_{1}$ such that $\left.u_{0}\right|_{\partial N}=f_{0}, u-u_{0} \in \mathcal{D}\left(\bar{A}_{\widetilde{\sigma}}\right)$ and

$$
\bar{A}_{\widetilde{\sigma}}\left[u-u_{0}, v\right]=-\int_{N} \tilde{\sigma} \nabla u_{0} \cdot \nabla v d x, \quad \text { for all } v \in \mathcal{D}\left(\bar{A}_{\tilde{\sigma}}\right)
$$

Then Cauchy data set of the finite energy solutions, denoted

$C_{f . e .}(\widetilde{\sigma})=\left\{\left(\left.u\right|_{\partial N},\left.\nu \cdot \widetilde{\sigma} \nabla u\right|_{\partial N}\right): u\right.$ is finite energy solution of $\left.\nabla \cdot \widetilde{\sigma} \nabla u=0\right\}$

coincides with $C_{\text {f.e. }}(\gamma)$. Using the above more general class of solutions, one can consider the non-zero frequency case,

$$
\nabla \cdot \widetilde{\sigma} \nabla u=\lambda u
$$

and show that the Cauchy data set of the finite energy solutions to the above equation coincides with the corresponding Cauchy data set for $\gamma$, cf. [32].

All of the above were obtained in dimensions $n \geq 3$. Kohn, Shen, Vogelius and Weinstein [55] have shown that the singular conductivity resulting from the same transformation also cloaks for electrostatics in two dimensions. Using estimates for the effect of small inclusions on the Dirichlet-Neumann map they gave precise estimates on how close one is to invisibility if the singular transformation is approximated by appropiate non-singular transformations.

\subsection{Quantum and Optical Shielding}

The uniqueness result of [96] applies more generally to the Schrödinger equation $-\Delta+q(x)$ when the potential $q(x)$ is assumed to be in $L^{\infty}$. In this case the DN map is defined by

$$
\Lambda_{q}(f)=\frac{\partial u}{\partial \nu}
$$

where $u$ solves the equation 


$$
(-\Delta+q) u=0, \quad \text { in } \Omega ;\left.\quad u\right|_{\partial \Omega}=f .
$$

We remark that the DN map is well defined only if 0 is not a Dirichlet eigenvalue of the Schrödinger equation. In the more general case we can define the set of Cauchy data

$$
C_{q}=\left\{\left(\left.u\right|_{\partial \Omega}, \frac{\partial u}{\partial \nu}\right) ; u \in H^{1}(\Omega) \text { solves }(-\Delta+q) u=0 \text { in } \Omega\right\} .
$$

The result of [96] states that $q$ is determined uniquely from $\Lambda_{q}$, or more generally $C_{q}$, in dimension three or larger. This was extended to $L^{n / 2}$ potentials in [63] and for conormal potentials having any singularity weaker than the delta function of a surface (see the precise result in [37]).

In particular case of this is the Helmholtz equation $-\Delta+k^{2} n(x)$ with a bounded isotropic index of refraction $n$.

In [37] we constructed a class of potentials or indices of refraction that shield any information contained in the region $D$, in other words the boundary information obtained outside the shielded region is the same as that the case of the potential 0 . These potentials behave like $q(x)=-C d(x, \partial D)^{-2-\epsilon}$ where $d$ denotes the distance to $\partial D$ and $C$ is a positive constant. As pointed out in [37], inside the region $D$ Schrödinger's cat could live for ever. From the point of view of quantum mechanics, $q$ represents a potential barrier so steep that no tunneling can occur. From the point of view of optics and acoustics, no sound waves or electromagnetic waves will penetrate, or emanate from, $D$. However, this construction should be thought of as shielding, not cloaking, since the potential barrier that shields that part of the potential within $D$ from boundary observation is itself detectable.

\section{Cloaking circa 2006}

\subsection{Developments in physics}

This brings us to the transformation-optics based proposals of [65, 80] for cloaking from observation by electromagnetic waves at positive frequency. 
One is interested in either scalar waves of the form $U(x, t)=u(x) e^{i k t}$, with $u$ satisfying the Helmholtz equation

$$
\left(\Delta+k^{2} n^{2}(x)\right) u(x)=\rho(x),
$$

where $\rho(x)$ represents sources that might be present or for time-harmonic electric and magnetic fields $\mathbf{E}(x, t)=E(x) e^{i k t}, \mathbf{H}(x, t)=H(x) e^{i k t}$, with $E, H$ satisfying Maxwell's equations,

$$
\nabla \times H=-i k \varepsilon E+J, \quad \nabla \times E=i k \mu H,
$$

where $J$ denotes any internal current present.

In three dimensions, if we start with the homogeneous, isotropic $\varepsilon_{0}, \mu_{0}$ on $B(0 ; 2)$ and push them forward by the "blowing up a point" map $F_{1}$ from (15), then they become inhomogeneous and anisotropic, identical to the conductivity tensor (18). Thus, they are nonsingular at each point of $N_{1}:=$ $B(0 ; 2) \backslash \bar{B}(0 ; 1)$, but as $r=|x| \longrightarrow 1^{+}$, two of the eigenvalues, associated to the angular directions, remain $\sim 1$, while the third, associated with the radial direction, is $\sim(r-1)^{2}$. Since the image of $F_{1}$ is just $N_{1}$, we chose the medium in the region to be cloaked, $N_{2}:=B(0 ; 1)$, by allowing $\varepsilon, \mu$ to be any smooth, nonsingular tensor there. This gives rise to what we call the single coating cloaking construction, to be physically implemented by layers of metamaterials on the exterior of the cloaking surface, $\Sigma=\partial N_{2}=\mathbb{S}^{2}$. We refer to $N:=N_{1} \cup N_{2} \cup \Sigma=B(0,2)$ as the cloaking device and the resulting specification of the material parameters on $N$ we denote by $\widetilde{\varepsilon}, \widetilde{\mu}$. In spherical coordinates, the representation of $\widetilde{\varepsilon}$ and $\widetilde{\mu}$ coincide with that of $\widetilde{\sigma}$ given in (18). Later, we will also describe the double coating construction, which corresponds to appropriately matched layers of metamaterials on both the outside and the inside of $\Sigma$.

Now, if one works exclusively on the open annulus $N_{1}$, the transformation $F_{1}$ is smooth and the chain rule, combined with (7), yields a one-to-one correspondence between solutions $(E, H)$ of Maxwell's equations (25) on $M_{1} \backslash$ $\{0\}=B(0 ; 2) \backslash 0$ and solutions $(\widetilde{E}, \widetilde{H})$ of Maxwell's equations on $N_{1}$, with internal current $\widetilde{J}$ arising from $\left.J\right|_{M_{1}}$ by an analogous transformation law. Thus, the boundary observations at $\partial N$ (or the scattering observations at infinity) seem to be unable to distinguish between the cloaking device $N$, with an object hidden from view in $N_{2}$, and the empty space of $M$. This 
is the level of justification that is presented in [80] and its sequels, [81, 26], where ray-tracing and numerical simulations on $N_{1}$ are given.

\section{$3.2 \quad$ Full-wave analysis}

Unfortunately, there is a serious problem with the above argument: it is insufficient to consider the waves merely outside of the cloaked region, i.e., on $N_{1}$; rather one needs to study the waves on all of $N$. Furthermore, a careful analysis should not ignore the fact that, since $\widetilde{\varepsilon}$ and $\widetilde{\mu}$ are degenerate at the cloaking surface $\Sigma$, without further conditions being imposed, the "waves" include some that are physically meaningless, even though of locally finite energy. (It is this degeneracy which causes the associated rays to go around the cloaked region, but its effect at the level of waves is what is crucial.) In fact, due to the degeneracy of $\widetilde{\varepsilon}$ and $\widetilde{\mu}$, the weighted $L^{2}$ space defined by the energy norm

$$
\|\widetilde{E}\|_{L^{2}\left(N,|\widetilde{g}|^{\frac{1}{2}} d x\right)}^{2}+\|\widetilde{H}\|_{L^{2}\left(N,|\widetilde{g}|^{\frac{1}{2}} d x\right)}^{2}=\int_{N}\left(\widetilde{\varepsilon}^{j k} \widetilde{E}_{j} \overline{\widetilde{E}_{k}}+\widetilde{\mu}^{j k} \widetilde{H}_{j} \overline{\widetilde{H}_{k}}\right) d x
$$

includes functions, which are not distributions, and for these the meaning of Maxwell's equations is problematic. Similar difficulties arise for the Helmholtz equation. To treat cloaking rigorously, one should consider the boundary measurements (or scattering data) of finite energy waves which also satisfy Maxwell's equations in some reasonable weak sense, such as the sense of distributions. This represents a strengthened version at positive frequency of Remark 2.4.

Analysis of cloaking from this more rigorous point of view was carried out in [32], which forms the basis for much of the discussion here. As it turns out, the insights gained from a careful analysis of the mathematical ideal cloaking construction arising from the singular transformation $F_{1}$, where these issues arise, leads to considerations that in fact improve the effectiveness of cloaking in more physically realistic approximations to the ideal [34].

\subsection{Physics on a Riemannian manifold}

Let us start with the cases of scalar optics or acoustics, governed in the case of isotropic media by the Helmholtz equation (24). In order to work with 
anisotropic media, we convert this to the Helmholtz equation with respect to a Riemannian metric $g$. Working in dimensions $n \geq 3$, we take advantage of the one-to-one correspondence (11) between (positive definite) contravariant 2-tensors of weight 1 and Riemannian metrics $g$. Let us consider the Helmholtz equation

$$
\left(\Delta_{g}+k^{2}\right) u=\rho
$$

where $\Delta_{g}$ is the Laplace-Beltrami operator associated with the Euclidian metric $g_{i j}=\delta_{i j}$. Under a smooth diffeomorphism $F$, the metric $g$ pushes forward to a metric $\widetilde{g}=F_{*} g$, and then, for $u=\widetilde{u} \circ F$, we have

$$
\left(\Delta_{g}+k^{2}\right) u=\rho \Longleftrightarrow\left(\Delta_{\widetilde{g}}+k^{2}\right) \widetilde{u}=\widetilde{\rho}
$$

where $\rho=\widetilde{\rho} \circ F$.

Next we consider the case when $F$ is not a smooth diffeomorphism, but $F=\left(F_{1}, F_{2}\right)$ as in $\S 2.2$.

Let $\tilde{f} \in L^{2}(N, d x)$ be a function such that $\operatorname{supp}(\widetilde{f}) \cap \Sigma=\emptyset$. We now give the precise definition of a finite energy solution for the Helmholtz equation. This definition applies for both the single and double coating constructions.

Definition 3.1 A measurable function $\widetilde{u}$ on $N$ is a finite energy solution of the Dirichlet problem for the Helmholtz equation on $N$,

$$
\begin{aligned}
& \left(\Delta_{\widetilde{g}}+k^{2}\right) \widetilde{u}=\widetilde{f} \text { on } N, \\
& \left.\widetilde{u}\right|_{\partial N}=\widetilde{h},
\end{aligned}
$$

if

$$
\begin{aligned}
& \widetilde{u} \in L^{2}\left(N,|\widetilde{g}|^{1 / 2} d x\right) ; \\
& \left.\widetilde{u}\right|_{N \backslash \Sigma} \in H_{l o c}^{1}(N \backslash \Sigma, d x) ; \\
& \int_{N \backslash \Sigma}|\widetilde{g}|^{1 / 2} \widetilde{g}^{i j} \partial_{i} \widetilde{u} \partial_{j} \widetilde{u} d x<\infty, \\
& \left.\widetilde{u}\right|_{\partial N}=\widetilde{h}
\end{aligned}
$$

and, for all $\widetilde{\psi} \in C^{\infty}(N)$ with $\left.\widetilde{\psi}\right|_{\partial N}=0$,

$$
\int_{N}\left[-\left(D_{\widetilde{g}}^{j} \widetilde{u}\right) \partial_{j} \widetilde{\psi}+k^{2} \widetilde{u} \widetilde{\psi}|\widetilde{g}|^{1 / 2}\right] d x=\int_{N} \widetilde{f}(x) \widetilde{\psi}(x)|\widetilde{g}|^{1 / 2} d x
$$


where $D_{\widetilde{g}}^{j} \widetilde{u}=|\widetilde{g}|^{1 / 2} \widetilde{g}^{i j} \partial_{i} u$ is defined as a Borel measure defining a distribution on $N$.

Note that the inhomogeneity $\widetilde{f}$ is allowed to have two components, $\widetilde{f}_{1}$ and $\widetilde{f}_{2}$, supported in the interiors of $N_{1}, N_{2}$, resp. The latter corresponds to an active object being rendered undetectable within the cloaked region. On the other hand, the former corresponds to an active object embedded within the metamaterial cloak itself, whose position apparently shifts in a predictable manner according to the transformation $F_{1}$; this phenomenon, which also holds for both spherical and cylindrical cloaking for Maxwell's equations, was later described and numerically modelled in the cylindrical setting, and termed the "mirage effect" [111].

Next we consider the relation of Maxwell's equations on $M$ and $N$. Recall that $F_{1}: M_{1} \backslash\{0\} \rightarrow N_{1}$ is singular and that $F_{2}: M_{2} \rightarrow N_{2}$ is the identity map and denote $\Gamma=\partial\left(\left(M_{1} \backslash\{0\}\right) \cup \partial M_{2}\right.$.

Theorem 3.2 ([32]) Let $u=\left(u_{1}, u_{2}\right):\left(M_{1} \backslash\{0\}\right) \cup M_{2} \rightarrow \mathbb{R}$ and $\widetilde{u}:$ $N \backslash \Sigma \rightarrow \mathbb{R}$ be measurable functions such that $u=\widetilde{u} \circ F$. Let $f=\left(f_{1}, f_{2}\right)$ : $\left(M_{1} \backslash\{0\}\right) \cup M_{2} \rightarrow \mathbb{R}$ and $\widetilde{f}: N \backslash \Sigma \rightarrow \mathbb{R}$ be $L^{2}$ functions, supported away from $\Gamma$ and $\Sigma$ such that $f=\tilde{f} \circ F$, and $\widetilde{h}: \partial N \rightarrow \mathbb{R}, h: \partial M_{1} \rightarrow \mathbb{R}$ be such that $h=\widetilde{h} \circ F_{1}$.

Then the following are equivalent:

1. The function $\widetilde{u}$, considered as a measurable function on $N$, is a finite energy solution to the Helmholtz equation (28) with inhomogeneity $\widetilde{f}$ and Dirichlet data $\widetilde{h}$ in the sense of Definition 3.1.

2. The function u satisfies

$$
\left(\Delta_{g}+k^{2}\right) u_{1}=f_{1} \quad \text { on } M_{1},\left.\quad u_{1}\right|_{\partial M_{1}}=h,
$$

and

$$
\left(\Delta_{g}+k^{2}\right) u_{2}=f_{2} \quad \text { on } M_{2},\left.\quad g^{j k} \nu_{j} \partial_{k} u_{2}\right|_{\partial M_{2}}=b,
$$

with $b=0$. Here $u_{1}$ denotes the continuous extension of $u_{1}$ from $M_{1} \backslash$ $\{0\}$ to $M_{1}$ 
Moreover, if $u$ solves (33) and (34) with $b \neq 0$, then the function $\widetilde{u}=u \circ F^{-1}$ : $N \backslash \Sigma \rightarrow \mathbb{R}$, considered as a measurable function on $N$, is not a finite energy solution to the Helmholtz equation.

As mentioned in $\S 1$, and detailed in [36], this result also describes a structure cloaking both passive objects and active sources for acoustic waves. Equivalent structures in the spherically symmetric case and with only cloaking of passive objects verified was considered later in $[22,28]$.

We point out that the Neumann boundary condition that appeared in (34) is a consequence of the fact that the coordinate transformation $F$ is singular on the cloaking surface $\Sigma$.

\subsection{Maxwell's equations}

In what follows, we treat Maxwell's equations in non-conducting and lossless media, that is, for which $\sigma=0$ and the components of $\varepsilon, \mu$ are real valued. Although somewhat suspect (presently, metamaterials are quite lossy), these are standard assumptions in the physical literature. We point out that Ola, Päivärinta and Somersalo [78] have shown that cloaking is not possible for Maxwell's equations with non-degenerate isotropic electromagnetic parameters.

We consider the electric and magnetic fields, $E$ and $H$, as differential 1-forms, given in some local coordinates by

$$
E=E_{j}(x) d x^{j}, \quad H=H_{j}(x) d x^{j} .
$$

For a smooth diffeomorphism $F$ and for a 1-form $E(x)=E_{1}(x) d x^{1}+E_{2}(x) d x^{2}+$ $E_{3}(x) d x^{3}$ we define the push-forward of $E$ in $F$, denoted $\widetilde{E}=F_{*} E$, by

$$
\begin{aligned}
\widetilde{E}(\widetilde{x}) & =\widetilde{E}_{1}(\widetilde{x}) d \widetilde{x}^{1}+\widetilde{E}_{2}(\widetilde{x}) d \widetilde{x}^{2}+\widetilde{E}_{3}(\widetilde{x}) d \widetilde{x}^{3} \\
& =\sum_{j=1}^{3}\left(\sum_{k=1}^{3}\left(D F^{-1}\right)_{j}^{k}(\widetilde{x}) E_{k}\left(F^{-1}(\widetilde{x})\right)\right) d \widetilde{x}^{j}, \quad \widetilde{x}=F(x) .
\end{aligned}
$$

A similar kind of transformation law is valid for 2-forms. We interpret the curl operator for 1-forms in $\mathbb{R}^{3}$ as being the exterior derivative, $d$. Maxwell's equations then have the form

$$
\operatorname{curl} H=-i k D+J, \quad \operatorname{curl} E=i k B
$$


where we consider the $D$ and $B$ fields and the external current $J$ (if present) as 2-forms. The constitutive relations are

$$
D=\varepsilon E, \quad B=\mu H,
$$

where the material parameters $\varepsilon$ and $\mu$ are linear maps mapping 1-forms to 2 -forms, i.e., are $(1,2)$ tensor fields.

Let $g$ be a Riemannian metric in $\Omega \subset \mathbb{R}^{3}$. Using the metric $g$, we define a specific permittivity and permeability by setting

$$
\varepsilon^{j k}=\mu^{j k}=|g|^{1 / 2} g^{j k} .
$$

This type of EM parameters were considered in [60] and have the same transformation laws as the case of Helmholtz equation or the conductivity equation.

To introduce the material parameters $\widetilde{\varepsilon}(x)$ and $\widetilde{\mu}(x)$ that make cloaking possible, we consider the singular map $F_{1}$ given by (15), the Euclidean metric on $N_{2}$ and $\widetilde{g}=F_{*} g$ in $N_{1}$. As before, and define the singular permittivity and permeability by the formula analogous to (17),

$$
\widetilde{\varepsilon}^{j k}=\widetilde{\mu}^{j k}= \begin{cases}|\widetilde{g}|^{1 / 2} \widetilde{g}^{j k} & \text { for } x \in N_{1}, \\ \delta^{j k} & \text { for } x \in N_{2} .\end{cases}
$$

We note that in $N_{2}$ one could define $\widetilde{\varepsilon}$ and $\widetilde{\mu}$ to be arbitrary smooth nondegenerate material parameters. For simplicity, we consider here only homogeneous material in the cloaked region $N_{2}$. As in the case of Helmholtz equations these material parameters, considered in $N_{1}$, are singular on $\Sigma$, requiring that what it means for fields $(\widetilde{E}, \widetilde{H})$ to form a solution to Maxwell's equations must be defined carefully.

\subsection{Definition of solutions of Maxwell equations}

Since the material parameters $\widetilde{\varepsilon}$ and $\widetilde{\mu}$ are again singular at the cloaking surface $\Sigma$, and keeping Remark 2.4 in mind, we need a careful formulation of the notion of a solution.

Definition 3.3 We say that $(\widetilde{E}, \widetilde{H})$ is a finite energy solution to Maxwell's equations on $N$,

$$
\nabla \times \widetilde{E}=i k \widetilde{\mu}(x) \widetilde{H}, \quad \nabla \times \widetilde{H}=-i k \widetilde{\varepsilon}(x) \widetilde{E}+\widetilde{J} \quad \text { on } N,
$$


if $\widetilde{E}, \widetilde{H}$ are one-forms and $\widetilde{D}:=\widetilde{\varepsilon} \widetilde{E}$ and $\widetilde{B}:=\widetilde{\mu} \widetilde{H}$ two-forms in $N$ with $L^{1}(N, d x)$-coefficients satisfying

$$
\begin{aligned}
\|\widetilde{E}\|_{L^{2}\left(N,|\widetilde{g}|^{1 / 2} d V_{0}(x)\right)}^{2} & =\int_{N} \widetilde{\varepsilon}^{j k} \widetilde{E}_{j} \overline{\widetilde{E}_{k}} d V_{0}(x)<\infty, \\
\|\widetilde{H}\|_{L^{2}\left(N,|\widetilde{g}|^{1 / 2} d V_{0}(x)\right)}^{2} & =\int_{N} \widetilde{\mu}^{j k} \widetilde{H}_{j} \overline{\widetilde{H}_{k}} d V_{0}(x)<\infty ;
\end{aligned}
$$

where $d V_{0}$ is the standard Euclidean volume, $(\widetilde{E}, \widetilde{H})$ is a classical solution of Maxwell's equations on a neighborhood $U \subset \bar{N}$ of $\partial N$ :

$$
\nabla \times \widetilde{E}=i k \widetilde{\mu}(x) \widetilde{H}, \quad \nabla \times \widetilde{H}=-i k \varepsilon(x) \widetilde{E}+\widetilde{J} \quad \text { in } U,
$$

and finally,

$$
\begin{aligned}
& \int_{N}((\nabla \times \widetilde{h}) \cdot \widetilde{E}-i k \widetilde{h} \cdot \widetilde{\mu}(x) \widetilde{H}) d V_{0}(x)=0, \\
& \int_{N}((\nabla \times \widetilde{e}) \cdot \widetilde{H}+\widetilde{e} \cdot(i k \widetilde{\varepsilon}(x) \widetilde{E}-\widetilde{J})) d V_{0}(x)=0
\end{aligned}
$$

for all 1-forms $\widetilde{e}, \widetilde{h}$ on $N$ having in the Euclidian coordinates components in $C_{0}^{\infty}(N)$.

Surprisingly, the finite energy solutions do not exist for generic currents. Below, we denote $M \backslash\{0\}=\left(M_{1} \backslash\{0\}\right) \cup M_{2}$.

Theorem 3.4 ([32]) Let $E$ and $H$ be 1-forms with measurable coefficients on $M \backslash\{0\}$ and $\widetilde{E}$ and $\widetilde{H}$ be 1-forms with measurable coefficients on $N \backslash \Sigma$ such that $\widetilde{E}=F_{*} E, \widetilde{H}=F_{*} H$. Let $J$ and $\widetilde{J}$ be 2-forms with smooth coefficients on $M \backslash\{0\}$ and $N \backslash \Sigma$, that are supported away from $\{0\}$ and $\Sigma$ such that $\widetilde{J}=F_{*} J$.

Then the following are equivalent:

1. The 1-forms $\widetilde{E}$ and $\widetilde{H}$ on $N$ satisfy Maxwell's equations

$$
\begin{aligned}
& \nabla \times \widetilde{E}=i k \widetilde{\mu}(x) \widetilde{H}, \quad \nabla \times \widetilde{H}=-i k \widetilde{\varepsilon}(x) \widetilde{E}+\widetilde{J} \quad \text { on } N, \\
& \nu \times\left.\widetilde{E}\right|_{\partial N}=f
\end{aligned}
$$

in the sense of Definition 3.3. 
2. The forms $E$ and $H$ satisfy Maxwell's equations on $M$,

$$
\begin{aligned}
& \nabla \times E=i k \mu(x) H, \quad \nabla \times H=-i k \varepsilon(x) E+J \quad \text { on } M_{1}, \\
& \nu \times\left. E\right|_{\partial M_{1}}=f
\end{aligned}
$$

and

$$
\nabla \times E=i k \mu(x) H, \quad \nabla \times H=-i k \varepsilon(x) E+J \quad \text { on } M_{2}
$$

with Cauchy data

$$
\nu \times\left. E\right|_{\partial M_{2}}=b^{e}, \quad \nu \times\left. H\right|_{\partial M_{2}}=b^{h}
$$

that satisfies $b^{e}=b^{h}=0$.

Moreover, if $E$ and $H$ solve (40), (41), and (42) with non-zero $b^{e}$ or $b^{h}$, then the fields $\widetilde{E}$ and $\widetilde{H}$ are not solutions of Maxwell equations on $N$ in the sense of Definition 3.3.

This can be interpreted as saying that the cloaking of active objects is difficult since, with non-zero currents present within the region to be cloaked, the idealized model leads to non-existence of finite energy solutions. The theorem says that a finite energy solution must satisfy the hidden boundary conditions

$$
\nu \times \widetilde{E}=0, \quad \nu \times \widetilde{H}=0 \quad \text { on } \partial N_{2} .
$$

Unfortunately, these conditions, which correspond physically to the so-called perfect electrical conductor (PEC) and perfect magnetic conductor (PMC) conditions, constitute an overdetermined set of boundary conditions for Maxwell's equations on $N_{2}$ (or, equivalently, on $M_{2}$ ). For cloaking passive objects, for which $J=0$, they can be satisfied, by fields which are identically zero in the cloaked region, but for generic $J$, including ones arbitrarily close to 0 , there is no solution.

The perfect, ideal cloaking devices in practice can only be approximated with a medium which material parameters approximate the degenerate parameters $\widetilde{\varepsilon}$ and $\widetilde{\mu}$. For instance, one can consider metamaterials built up using periodic structures whose effective material parameters approximate $\widetilde{\varepsilon}$ and $\widetilde{\mu}$. Thus the question of when the solutions exists in a reasonable sense is directly related to the question of which approximate cloaking devices can be built 
in practice. We note that if $E$ and $H$ solve (40), (41), and (42) with nonzero $b^{e}$ or $b^{h}$, then the fields $\widetilde{E}$ and $\widetilde{H}$ can be considered as solutions of a non-homogeneous Maxwell equations on $N$ in the sense of Definition 3.3.

$$
\nabla \times \widetilde{E}=i k \widetilde{\mu}(x) \widetilde{H}+\widetilde{K}_{\text {surf }}, \quad \nabla \times \widetilde{H}=-i k \widetilde{\varepsilon}(x) \widetilde{E}+\widetilde{J}+\widetilde{J}_{\text {surf }} \quad \text { on } N
$$

where $\widetilde{K}_{\text {surf }}$ and $\widetilde{J}_{\text {surf }}$ are magnetic and surface currents supported on $\Sigma$. If we include a PEC lining on the inner side of $\Sigma$, the solution for the given boundary value $f$ is the one where $\widetilde{K}_{\text {surf }}=0$ and $\widetilde{J}_{\text {surf } f}$ is possibly non-zero and in the case of PMC lining the solution is the one with $\widetilde{J}_{\text {surf }}=0$. If we are building an approximate cloaking device with metamaterials, effective constructions could be done in such a way that the material approximates a cloaking material with PEC or PMC lining. We will discuss this question in detail in the next section in the context of cylindrical cloaking. In that case, adding a special physical surface on $\Sigma$ improves significantly the behavior of approximate cloaking devices; without this kind of lining the fields blow up. This suggests that experimentalists building cloaking devices should consider first what kind of cloak with well-defined solutions they would like to approximate. Indeed, building a device where solutions behave nicely is probably easier than building one with huge oscillations of the fields.

As an alternative, one can modify the basic construction by using a double coating. Mathematically, this corresponds to using an $F=\left(F_{1}, F_{2}\right)$ with both $F_{1}, F_{2}$ singular, which gives rise to a singular Riemannian metric which degenerates in the same way as one approaches $\Sigma$ from both sides. Physically, the double coating construction corresponds to surrounding both the inner and outer surfaces of $\Sigma$ with appropriately matched metamaterials.

\section{Cylindrical cloaking, approximate cloaking and the SHS lining}

In the following we change the geometrical situation where we do our considerations, and redefine the meaning of the used notations.

We consider next an infinite cylindrical domain. Below, $B_{2}(0, r) \subset \mathbb{R}^{2}$ is Euclidian disc with center 0 and radius $r$. The cloaking device $N$ is in the cylindrical case the infinite cylinder $N=B_{2}(0,2) \times \mathbb{R}$ that contains the subsets $N_{1}=\left(B_{2}(0,2) \backslash \bar{B}_{2}(0,1)\right) \times \mathbb{R}$, and $N_{2}=B_{2}(0,1) \times \mathbb{R}$. We will consider 
observations on the surface $\partial N$. Moreover, let $M$ be the disjoint union of $M_{1}=B_{2}(0,2) \times \mathbb{R}$ and $M_{2}=B_{2}(0,1) \times \mathbb{R}$. Finally, in this section the cloaking surface is $\Sigma=\partial B_{2}(0,1) \times \mathbb{R}$, and we denote $L=\{(0,0)\} \times \mathbb{R} \subset M_{1}$. Next, we consider cylindrical coordinates, $(r, \theta, z) \mapsto(r \cos \theta, r \sin \theta, z)$. The singular coordinate transformation in these coordinates is the map $F: M \backslash L \rightarrow N \backslash \Sigma$, given by

$$
\begin{aligned}
& F(r, \theta, z)=\left(1+\frac{r}{2}, \theta, z\right), \quad \text { on } M_{1} \backslash L, \\
& F(r, \theta, z)=(r, \theta, z), \quad \text { on } M_{2} .
\end{aligned}
$$

Again, let $g$ be the Euclidian metric on $M$, that is, on both components $M_{1}$ and $M_{2}$, and $\varepsilon=1$ and $\mu=1$ be homogeneous material parameters in $M$. Using map $F$ we define $\widetilde{g}=F_{*} g$ in $N \backslash \Sigma$ and define the corresponding material parameters $\widetilde{\varepsilon}$ and $\widetilde{\mu}$ as in formula (35). By locally finite energy solutions of Maxwell's equations on $N$ we will mean locally integrable oneforms $\widetilde{E}$ and $\widetilde{H}$ satisfying in all bounded open sets $N^{\prime} \subset N$ the conditions analogous to Definition 3.3. We recall that $\widetilde{E}, \widetilde{H}$ are finite energy solutions in a bounded domain $N^{\prime}$ means in particular that those are 1-forms and $\widetilde{D}=\widetilde{\varepsilon} \widetilde{E}, \widetilde{B}=\widetilde{\mu} \widetilde{H}$ are 2-forms with $L^{1}\left(N^{\prime}, d x\right)$-coefficients. We note that in the cylindrical cloaking $\widetilde{\varepsilon}$ and $\widetilde{\mu}$ are not any more bounded, and they have in $N_{1}$ in cylindrical coordinates the representation

$$
\widetilde{\varepsilon}=\widetilde{\mu}=\left(\begin{array}{ccc}
(r-1) & 0 & 0 \\
0 & (r-1)^{-1} & 0 \\
0 & 0 & 4(r-1)
\end{array}\right), \quad 1<r<2 .
$$

Let us denote by $\zeta=\partial_{z}$ the vertical vector field in $\mathbb{R}^{3}$.

We will consider 1-forms $E$ and $H$ on $M$ and $\widetilde{E}$ and $\widetilde{H}$ on $N$ that satisfy $\widetilde{E}=F_{*} E$ and $\widetilde{H}=F_{*} H$ on $N \backslash \Sigma$. For simplicity, we will consider the case when

$$
\begin{aligned}
& \widetilde{E}=0 \text { and } \widetilde{H}=0 \text { in } N_{2}, \quad \text { or equivalently, } \\
& E=0 \text { and } H=0 \text { in } M_{2} .
\end{aligned}
$$

This corresponds to the case when the cloaked region $N_{2}$ is dark. In this case Theorem 7.1 in [32] yields the following result: 
Theorem 4.1 Let $E$ and $H$ be 1-forms on $M$ and $\widetilde{E}$ and $\widetilde{H}$ be 1-forms on $N$ such that $\widetilde{E}=F_{*} E$ and $\widetilde{H}=F_{*} H$ on $N \backslash \Sigma$. Assume that (44) is valid and that $\widetilde{E}$ and $\widetilde{H}$ are locally finite energy solution of Maxwell's equations on $N$. Then the forms $E$ and $H$ are classical solutions to Maxwell's equations on $M$ and the restrictions on the line $L \subset M_{1}$,

$$
b_{1}^{e}=\left.\zeta \cdot E\right|_{L}, \quad b_{1}^{h}=\left.\zeta \cdot H\right|_{L}
$$

must satisfy $b_{1}^{e}=0$ and $b_{1}^{h}=0$.

This results implies that if we impose on some boundary condition on the exterior boundary of $N_{1}$, e.g., the electric boundary condition $\nu \times\left.\widetilde{E}\right|_{\partial B_{2}(0,2) \times \mathbb{R}}=$ $f$, the locally finite energy solutions on $N$ exists only if Maxwell's equations

$$
\begin{aligned}
& \nabla \times E=i k \mu(x) H, \quad \nabla \times H=-i k \varepsilon(x) E \quad \text { on } M_{1}, \\
& \nu \times\left. E\right|_{\partial M_{1}}=f
\end{aligned}
$$

have a solution which restrictions (45) on the line $L$ vanish. So, with generic electric boundary value $f$ the locally locally finite energy solution does not exists.

Again, there is a remedy for this obstruction for cloaking. Using transformation rule (7) one can observe for the locally finite energy solutions that in Euclidian coordinates on $N_{1} \subset \mathbb{R}^{3}$ the $\theta$-component of the fields $\widetilde{H}$ and $\widetilde{E}$ vanish on $\Sigma$. Motivated by this we impose the soft-and-hard surface (SHS) boundary condition on the cloaking surface. This can be considered by attaching a soft-and-hard surface on the inside of the cloaking material. In classical terms, an SHS condition on a surface $\Sigma[40,47]$ is

$$
\left.\eta \cdot E\right|_{\Sigma}=0 \text { and }\left.\eta \cdot H\right|_{\Sigma}=0
$$

where $\eta=\eta(x)$ is some tangential vector field on $\Sigma$, that is, $\eta \cdot \nu=0$. In other words, the part of the tangential component of the electric field $E$ that is parallel to $\eta$ vanishes, and the same is true for the magnetic field $H$. This was originally introduced in antenna design and can be physically realized by having a surface with thin parallel gratings filled with dielectric material $[47,48,67,40]$. Here, we consider this boundary condition when $\eta$ is the vector field $\eta=\partial_{\theta}$, that is, the angular vector field that is tangential to $\Sigma$.

For simplicity, let us consider a case where the cloaked region $N_{2}$ is replaced by an obstacle, and on the boundary of the obstacle we have the SHS-boundary condition. Thus the field is defined only in the domain $N_{1}$. 
Definition 4.2 We say that the 1-forms $\widetilde{E}$ and $\widetilde{H}$ are locally finite energy solutions of Maxwell's equations on $N_{1}$ with soft-and-hard surface (SHS) boundary conditions on $\Sigma$,

$$
\begin{aligned}
\nabla \times \widetilde{E} & =i k \widetilde{\mu}(x) \widetilde{H}, \quad \nabla \times \widetilde{H}=-i k \widetilde{\varepsilon}(x) \widetilde{E}+\widetilde{J} \quad \text { on } N_{1}, \\
\left.\eta \cdot \widetilde{E}\right|_{\Sigma} & =0,\left.\quad \eta \cdot \widetilde{H}\right|_{\Sigma}=0,
\end{aligned}
$$

if $\widetilde{E}$ and $\widetilde{H}$ are 1-forms and $\widetilde{\varepsilon} \widetilde{E}$ and $\widetilde{\mu} \widetilde{H}$ are 2-forms on $N_{1}$ with coefficients in $L_{\text {loc }}^{1}\left(N_{1}, d x\right)$ satisfying $\|\widetilde{E}\|_{L^{2}\left(S,|\widetilde{g}|^{1 / 2} d V_{0}\right)}^{2}<\infty,\|\widetilde{H}\|_{L^{2}\left(S,|\widetilde{g}|^{1 / 2} d V_{0}\right)}^{2}<\infty$ for all open and bounded subsets $S \subset N_{1}$, and

$$
\begin{aligned}
& \int_{N_{1}}((\nabla \times \widetilde{h}) \cdot \widetilde{E}-i k \widetilde{h} \cdot \widetilde{\mu}(x) \widetilde{H}) d V_{0}(x)=0, \\
& \int_{N_{1}}((\nabla \times \widetilde{e}) \cdot \widetilde{H}+\widetilde{e} \cdot(i k \widetilde{\varepsilon}(x) \widetilde{E}-\widetilde{J})) d V_{0}(x)=0,
\end{aligned}
$$

for all $\widetilde{e}, \widetilde{h}$ that are 1 -forms having coefficients in $C^{\infty}\left(\bar{N}_{1}\right)$, supported in a bounded set, vanishing near $\partial N$, and satisfying

$$
\left.\eta \cdot \widetilde{e}\right|_{\Sigma}=0,\left.\quad \eta \cdot \widetilde{h}\right|_{\Sigma}=0 .
$$

The following invisibility result holds:

Theorem 4.3 ([32]) Let $E$ and $H$ be 1-forms with measurable coefficients on $M_{1}$ and $\widetilde{E}$ and $\widetilde{H}$ be 1-forms with measurable coefficients on $N_{1}$ such that $E=F^{*} \widetilde{E}, H=F^{*} \widetilde{H}$. Let $J$ and $\widetilde{J}$ be Q2-forms with smooth coefficients on $M_{1}$ and $N_{1}$, that are supported away from $L$ and $\Sigma$ such that $J=F^{*} \widetilde{J}$ in $N_{1}$. Then the following are equivalent:

1. On $N_{1}$, the 1-forms $\widetilde{E}$ and $\widetilde{H}$ satisfy Maxwell's equations with SHS boundary conditions in the sense of Definition 4.2.

2. On $M_{1}$, the forms $E$ and $H$ are classical solutions of Maxwell's equations,

$$
\begin{aligned}
& \nabla \times E=i k \mu(x) H, \quad \text { in } M_{1} \\
& \nabla \times H=-i k \varepsilon(x) E+J, \quad \text { in } M_{1} .
\end{aligned}
$$


This result implies that when the surface $\Sigma$ is lined with a material implementing the SHS boundary condition, the locally finite energy solutions exist for all incoming waves.

How then the non-existence result can be interpreted? Let us consider the situation when a metamaterial coating only approximates the ideal invisibility coating. More precisely, for $1<R<2$, consider an infinite cylinder in $\mathbb{R}^{3}$ given, in cylindrical coordinates, by $N_{2}^{R}=\{r<R\}$. On $N_{2}^{R}$ we choose the metric to be Euclidean, so that the corresponding permittivity and permeability, $\varepsilon_{0}$ and $\mu_{0}$, are homogeneous and isotropic. In $N_{1}^{R}=N \backslash N_{2}^{R}$, we take the Riemannian metric $\widetilde{g}$ and the corresponding permittivity and permeability $\widetilde{\varepsilon}$ and $\widetilde{\mu}$ defined in (35) above. This yields that the approximate coating has the finite anisotropy ratio,

$$
L_{R}:=\max _{1 \leq j, k \leq 3} \sup _{x \in N} \frac{\lambda_{j}(x)}{\lambda_{k}(x)}
$$

where $\lambda_{j}(x), j=1,2,3$, are the eigenvalues of $\widetilde{\varepsilon}(x)$ or $\widetilde{\mu}(x)$. Thus Maxwell's equation are defined in the approximate coating in the classical way. We call the domain $N$ with the approximate $\widetilde{\varepsilon}$ and $\widetilde{\mu}$ the approximate cloaking device.

Using the approximate coating we considered the scattering problem where a plane wave hits to approximate cloaking device when the cloaked region $N_{2}^{R}$ is filled with a homogenous isotropic material, $\varepsilon=\mu=\delta^{j k}$ and $\Sigma$ contains no lining. Then the total fields $\widetilde{E}^{R}$ and $\widetilde{H}^{R}$ and the total fluxes $\widetilde{D}^{R}$ and $\widetilde{B}^{R}$ converge when $R \rightarrow 1$, in the sense of distributions,

$$
\begin{aligned}
\lim _{R \rightarrow 1^{+}} \widetilde{E}^{R} & =\widetilde{E}_{\text {lim }}, \quad \lim _{R \rightarrow 1^{+}} \widetilde{H}^{R}=\widetilde{H}_{\text {lim }}, \\
\lim _{R \rightarrow 1^{+}} \widetilde{D}^{R} & =\widetilde{\varepsilon} \widetilde{E}_{\text {lim }}-\frac{1}{i k} \widetilde{J}_{\text {surf }}, \\
\lim _{R \rightarrow 1^{+}} \widetilde{B}^{R} & =\widetilde{\mu} \widetilde{H}_{\text {lim }}+\frac{1}{i k} \widetilde{K}_{\text {surf }},
\end{aligned}
$$

where $\widetilde{E}_{l i m}$ and $\widetilde{H}_{l i m}$ are measurable functions and $J_{\text {surf }}$ and $\widetilde{K}_{\text {surf }}$ are deltadistributions supported on $\Sigma$ multiplied with smooth 2-forms corresponding to tangential currents on $\Sigma$. Thus when the approximated coating approaches the ideal, that is, $R \rightarrow 1^{+}$, we obtain on the limit the equations

$$
\begin{aligned}
& \nabla \times \widetilde{E}_{l i m}=i \omega \widetilde{B}_{l i m}+\widetilde{K}_{\text {surf }}, \quad \nabla \times \widetilde{H}_{l i m}=-i \omega \widetilde{D}_{l i m}+\widetilde{J}_{\text {surf }}, \\
& \widetilde{D}_{l i m}=\widetilde{\varepsilon} \widetilde{E}_{l i m}, \quad \widetilde{B}_{l i m}=\widetilde{\mu} \widetilde{H}_{l i m} .
\end{aligned}
$$


The equations (50) were introduced in [32]. In numerical simulations in [33] we considered scattering of a TE-polarized plane wave from a cylindrical cloaking device with approximate coating in two cases: when the cloaked region is filled with a homogeneous isotropic material, and when inside the coating there is a soft-and-hard surface. See Fig. 4.
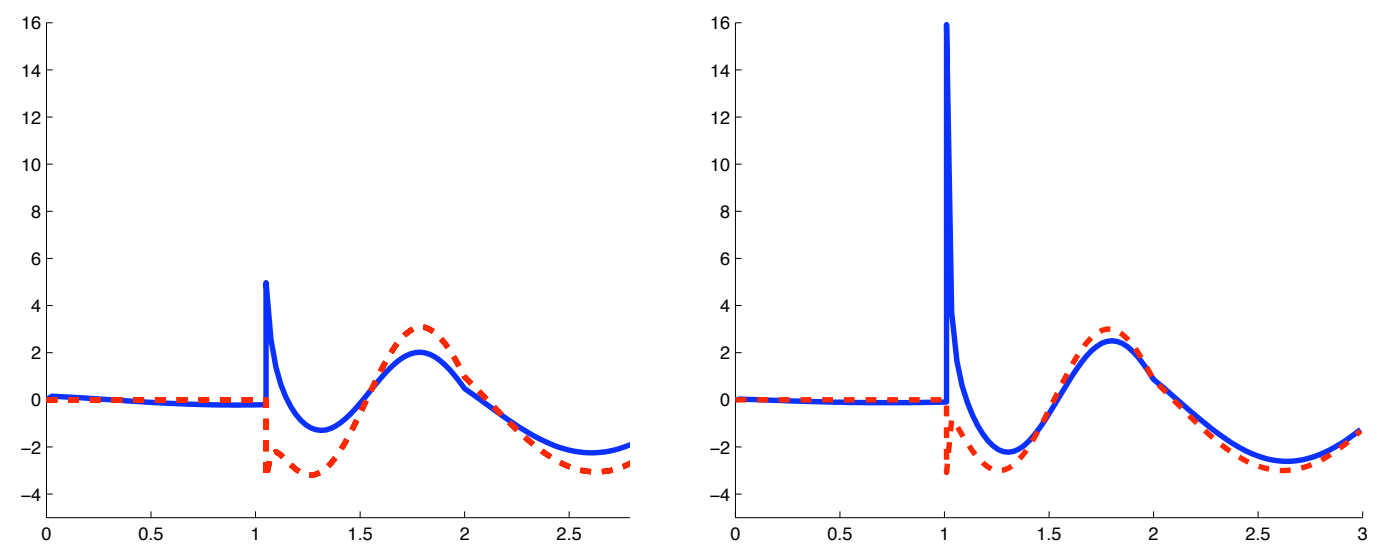

Figure 4: The real part of the $y$-component of the total $B$-field on the line $\{(x, 0,0): x \in[0,3]\}$ when a TE-plane wave scatters from an approximate cloaking device. Blue solid curve is the field with no physical lining at $\{r=$ $R\}$. Red dashed curve is the field with SHS lining on $\{r=R\}$. In the left figure, $R=1.05$ and the maximal anisotropy ratio is $L_{R}=1600$. In the right figure, $R=1.01$ and the maximal anisotropy ratio is $L_{R}=40,000$.

In Fig. 4, the development of the delta-distribution on the cloaking surface, i.e., the blow up of the fields as the approximate cloak improves, can be clearly observed. Very similar behavior in the absence of a lining was previously obtained by Ruan, Yan, Neff and Qiu [87] by scattering methods. They showed that, in the case of cylindrical cloaking, with no internal currents and no lining, the fields for the truncated cloak converges at best logarithmically to the fields for the ideal cloak. Similar results for Helmholtz have now also been reported by Kohn, et al., [54].

Since the metamaterials used to implement cloaking are based on effective medium theory, the resulting large variation in $D$ and $B$ poses a challenge to the suitability of field-averaged characterizations of $\varepsilon$ and $\mu$ [92]. (We note in passing that there still are many open questions in the mathematically 
rigorous effective medium theory for materials that might implement such parameters. For recent results directly applicable to metamaterials used for cloaking, see, e.g., [56], while closely related issues concerning negative index materials are in $[9,10,11,12,13]$.)

The approximate cloaking is also significantly improved by the SHS lining in the sense that both the far field of the scattered wave is significantly reduced and the blow up of $D$ and $B$ prevented. For instance, in the simulation presented in figure 4 with $R=1.01$ the $L^{2}$-norm of the far field pattern with the SHS lining was only $2 \%$ of the far field without the SHS lining, see [33].

\section{Electromagnetic wormholes}

We describe in this section another application of transformation optics which consists in "blowing" up a line rather than a point. In [33, 35] a blueprint is given for a device that would function as an invisible tunnel, allowing EM waves to propagate from one region to another, with only the ends of the tunnel being visible. Such a device, making solutions of Maxwell's equations behave as if the topology of $\mathbb{R}^{3}$ has been modified by the attachment of a handle, is analogous to an Einstein-Rosen wormhole [29], and so we refer to this construction as an electromagnetic wormhole.

We first give a general description of the electromagnetic wormhole. Consider first as in Fig. 5 a 3-dimensional wormhole manifold (or handlebody) $M=$ $M_{1} \# M_{2}$ where the components

$$
\begin{aligned}
& M_{1}=\mathbb{R}^{3} \backslash(B(O, 1) \cup B(P, 1)), \\
& M_{2}=\mathbb{S}^{2} \times[0,1]
\end{aligned}
$$

are glued together smoothly.

An optical device that acts as a wormhole for electromagnetic waves at a given frequency $k$ can be constructed by starting with a two-dimensional finite cylinder

$$
T=\mathbb{S}^{1} \times[0, L] \subset \mathbb{R}^{3}
$$

and taking its neighborhood $K=\left\{x \in \mathbb{R}^{3}: \operatorname{dist}(x, T) \leq \rho\right\}$, where $\rho>0$ is small enough and $N=\mathbb{R}^{3} \backslash K$. Let us put on $\partial K$ the SHS boundary condition and cover $K$ with "invisibility cloaking material", that in the boundary 


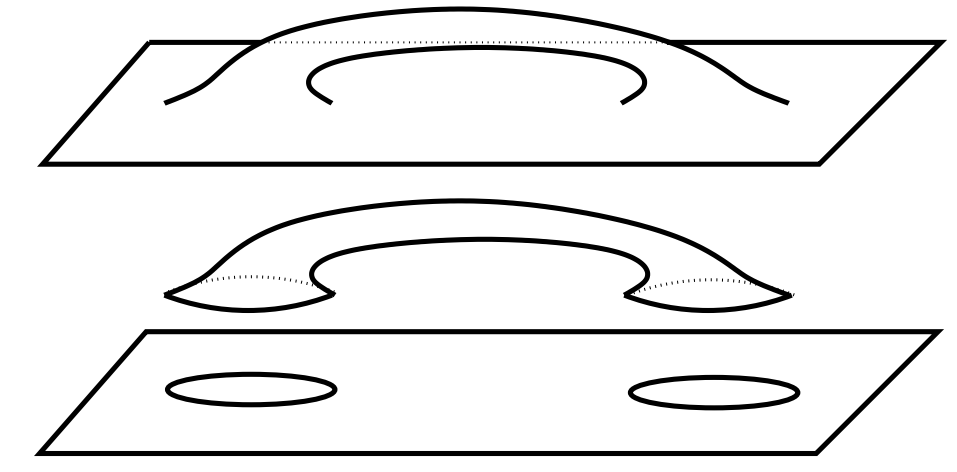

Figure 5: A two dimensional schematic figure of wormhole construction by gluing surfaces. Note that the components of the artificial wormhole construction are three dimensional.

normal coordinates around $K$ has the same representation as $\widetilde{\varepsilon}$ and $\widetilde{\mu}$ when cloaking an infinite cylinder. Finally, let

$$
U=\left\{x \in \mathbb{R}^{3}: \operatorname{dist}(x, K)>1\right\}
$$

and note that $\widetilde{\varepsilon}, \widetilde{\mu}$ are equal to $\delta^{j k}$ in $U$. The set $U$ can be considered both a subset of $N \subset \mathbb{R}^{3}$ and a part of the abstract wormhole manifold $M, U \subset M_{1}$. Then, for currents supported in $U$, all measurements of the electromagnetic fields in $U \subset M$ and $U \subset N$ coincide; that is, waves on the wormhole device $(N, \widetilde{\varepsilon}, \widetilde{\mu})$ in $\mathbb{R}^{3}$ behave as if they were propagating on the abstract handlebody space $M$. This of course produces global effects on the waves passing through the device, contrary to the claim in $[85, \S 2]$.

In Figures 6(a) and 6(b) we give ray-tracing simulations in and near the wormhole. The obstacle in the figures is $K$, and the metamaterial corresponding to $\widetilde{\varepsilon}$ and $\widetilde{\mu}$, through which the rays travel, is not shown.

We now give a more precise description of an electromagnetic wormhole. Let us start by making two holes in $\mathbb{R}^{3}$, say by removing the open unit ball $B_{1}=B(O, 1)$, and also the open ball $B_{2}=B(P, 1)$, where $P=(0,0, L)$ is a point on the $z$-axis with $L>3$, so that $\overline{B_{1}} \cap \overline{B_{2}}=\emptyset$. The region so obtained, $M_{1}=\mathbb{R}^{3} \backslash\left(B_{1} \cup B_{2}\right)$, equipped with the standard Euclidian metric 

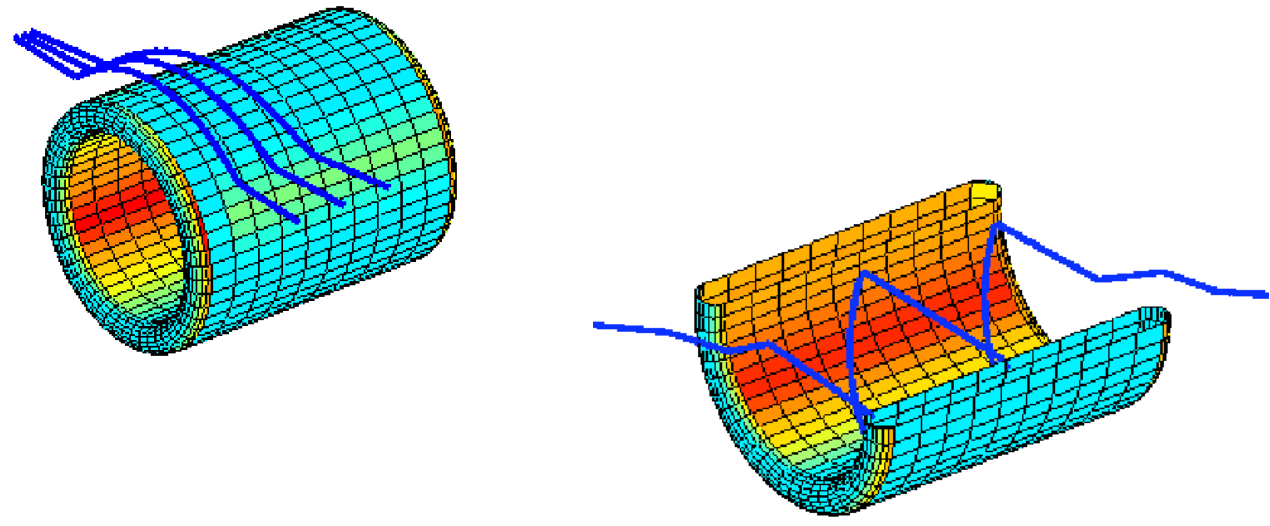

Figure 6: (a) Rays travelling outside.

(b) A ray travelling inside.
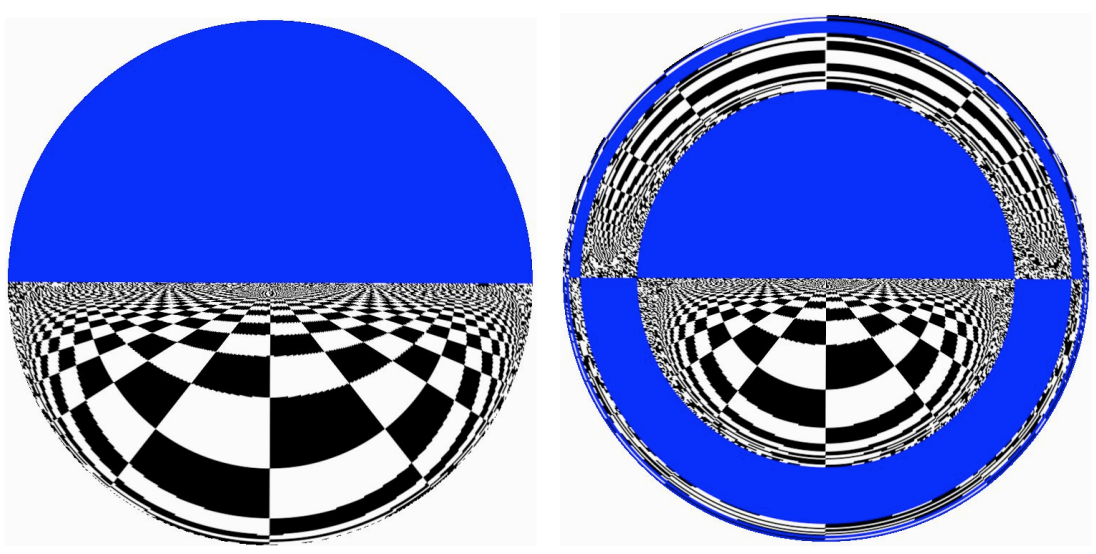

Figure 7: Ray tracing simulations of views through the bores of two wormholes. The distant ends are above an infinite chess board under a blue sky. On left, $L<<1$; on right, $L \approx 1$. Note that blue is used for clarity; the wormhole construction should be considered essentially monochromatic, for physical rather than mathematical reasons. 
$g_{0}$ and with $\gamma_{1}=\{(0,0, z): 1 \leq z \leq L-1\}$, is the first component $M_{1}$ of the wormhole manifold. Note that $M_{1}$ is a 3 -dimensional manifold with boundary $\partial M_{1}=\partial B_{1} \cup \partial B_{2}$. i.e., $\partial M_{1}$ can be considered as $\mathbb{S}_{1}^{2} \cup \mathbb{S}_{2}^{2}$, where we will use $\mathbb{S}_{*}^{2}$ to denote various copies of the two-dimensional unit sphere.

The second component of the wormhole manifold is a 3 -dimensional cylinder, $M_{2}=\mathbb{S}^{2} \times[0,1]$, with boundary $\partial M_{2}=\left(\mathbb{S}^{2} \times\{0\}\right) \cup\left(\mathbb{S}^{2} \times\{1\}\right):=\mathbb{S}_{3}^{2} \cup \mathbb{S}_{4}^{2}$. We take $\gamma_{2}=\{N P\} \times[0,1]$, where $N P$ denotes an arbitrary point in $\mathbb{S}^{2}$, say the North Pole. We initially equip $M_{2}$ with the product metric, but several variations on this basic design are possible, having somewhat different possible applications which will be mentioned below.

One can form a handlebody by gluing together the component $\mathbb{S}_{1}^{2}$ of the boundary $\partial M_{1}$ with the lower end boundary component $\mathbb{S}_{3}^{2}$ of $M_{2}$ and the component $\mathbb{S}_{2}^{2}$ of the boundary $\partial M_{1}$ with the upper end $\mathbb{S}_{4}^{2}$. In doing so we glue the point $(0,0,1) \in \partial B(O, 1)$ with the point $N P \times\{0\}$ and the point $(0,0, L-1) \in \partial B(P, 1)$ with the point $N P \times\{1\}$. Note that in this construction, $\gamma_{1}$ and $\gamma_{2}$ correspond to two nonhomotopic rays connecting $(0,0,1) \sim N P \times\{0\}$ to $(0,0, L-1) \sim N P \times\{1\}$.

Let us denote in cylindrical coordinates $N_{2}=\{(r, \theta, z):|r|<1, z \in$ $[0, L]\} \cap N$ and $N_{1}=N \backslash N_{2}$ and consider singular transformations $F_{j}$ : $M_{j} \backslash \gamma_{j} \longrightarrow \mathbb{R}^{3}, j=1,2$, whose images are $N_{1}, N_{2}$, correspondingly, see [35] for details. For instance, the map $F_{1}$ can be chosen so that it keeps the $\theta$-coordinate the same and maps $(r, z)$ coordinates by $f_{1}:(r, z) \rightarrow\left(r^{\prime}, z^{\prime}\right)$. In the Figure 8 the map $f_{1}$ is visualized.

Possible applications of electromagnetic wormholes (with varying degrees of likelihood of realization!), when the metamaterials technology has sufficiently progressed, include invisible optical cables, 3D video displays, scopes for MRI-assisted medical procedures, and beam collimation. For the last two, one needs to modify the design by changing the metric $g_{2}$ on $M_{2}=\mathbb{S}^{2} \times[0,1]$. By flattening the metric on $\mathbb{S}^{2}$ so that the antipodal point $S P$ (the south pole) to $N P$ has a neighborhood on which the metric is Euclidian, the axis of the tunnel $N_{2}$ will have a tubular neighborhood on which $\varepsilon, \mu$ are constant isotropic and hence can be allowed to be empty space, allowing for passage of instruments. On the other hand, if we use a warped product metric on $M_{2}$, corresponding to $\mathbb{S}^{2} \times\{z\}$ having the metric of the sphere of radius $r(z)$ for an appropriately chosen function $r:[0,1] \longrightarrow \mathbb{R}_{+}$, only rays that travel through $N_{2}$ almost parallel to the axis can pass all the way through, with 

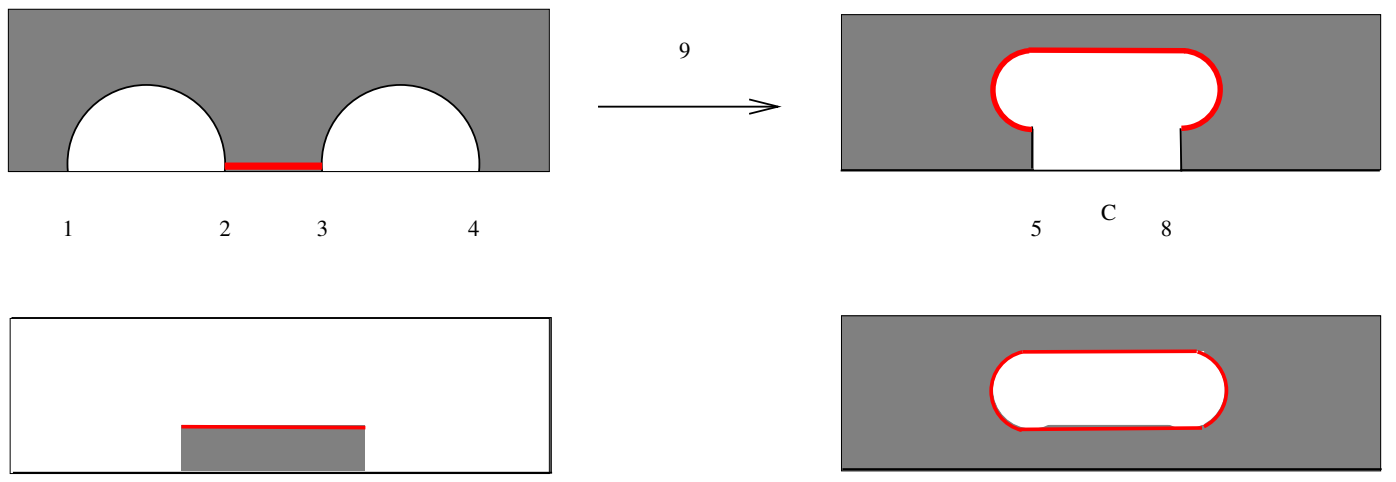

A

B

Figure 8: Above: A schematic figure of $f_{1}$, representing $F_{1}$, in the $(r, z)$ plane. Its image $P$ corresponds to $N_{1}$ in $(r, z)$ coordinates. Below: The sets $Q$ and $R$ correspond to $N_{2}$ and $N$. In the figure, $R=Q \cup P$ which corresponds to $N=N_{1} \cup N_{2}$ in $\mathbb{R}^{3}$.

others being returned to the end from which they entered.

\section{A general framework: singular transformation optics}

Having seen how cloaking based on blowing up a point or blowing up a line can be rigorously analyzed, we now want to explore how more general optical devices can be described using the transformation rules satisfied by $n,(\rho, \lambda), \varepsilon$ and $\mu$. This point of view has been advocated by J. Pendry and his collaborators, and given the name transformation optics [101]. As discussed earlier, under a nonsingular changes of variables $F$, there is a one-to-one correspondence between solutions $\widetilde{u}$ of the relevant equations for the transformed medium and solutions $u=\widetilde{u} \circ F$ of the original medium. However, when $F$ is singular at some points, as is the case for cloaking and the wormhole, we have shown how greater care needs to be taken, not just for the sake of mathematical rigor, but to improve the cloaking effect for more physically realistic approximations to the ideal material parameters. Cloaking and wormholes can be considered as merely starting points for what might be termed singular transformation optics, which, combined with the rapidly 
developing technology of metamaterials, opens up entirely new possibilities for designing devices having novel effects on acoustic or electromagnetic wave propagation. Other singular transformation designs in $2 \mathrm{D}$ that rotate waves within the cloak [20], concentrate waves [84] or act as beam splitters [85] have been proposed. Analogies with phenomena in general relativity have been proposed in [66] as a source of inspiration for designs.

We formulate a general approach to the precise description of the ideal material parameters in a singular transformation optics device, $N \subset \mathbb{R}^{3}$, and state a "metatheorem", analogous to the results we have seen above, which should, in considerable generality, give an exact description of the electromagnetic waves propagating through such a device. However, we wish to stress that, as for cloaking [32] and the wormhole [33, 35], actually proving this "result" in particular cases of interest, and determining the hidden boundary conditions, may be decidedly nontrivial.

A general framework for considering ideal mathematical descriptions of such designs is as follows: Define a singular transformation optics (STO) design as a triplet $(\mathcal{M}, \mathcal{N}, \mathcal{F})$, consisting of:

(i) An STO manifold, $\mathcal{M}=(M, g, \gamma)$, where $M=\left(M_{1}, \ldots, M_{k}\right)$, the disjoint union of $n$-dimensional Riemannian manifolds $\left(M_{j}, g_{j}\right)$, with or without boundary, and (possibly empty) submanifolds $\gamma_{j} \subset \operatorname{int} M_{j}$, with $\operatorname{dim} \gamma_{j}=0$ or 1 ;

(ii) An STO device, $\mathcal{N}=(N, \Sigma)$, where $N=\bigcup_{j=1}^{k} N_{j} \subset \mathbb{R}^{n}$ and $\Sigma=$ $\bigcup_{j=1}^{k} \Sigma_{j}$, with $\Sigma_{j}$ a (possibly empty) hypersurface in $N_{j}$; and

(iii) A singular transformation $\mathcal{F}=\left(F_{1}, \ldots, F_{k}\right)$, with each $F_{j}: M_{j} \backslash \gamma_{j} \longrightarrow N_{j} \backslash \Sigma_{j}$ a diffeomorphism.

Note that $N$ is then equipped with a singular Riemannian metric $\widetilde{g}$, with $\left.\widetilde{g}\right|_{N_{j}}=\left(F_{j}\right)_{*}\left(g_{j}\right)$, in general degenerate on $\Sigma_{j}$. Reasonable conditions need to be placed on the Jacobians $D F_{j}$ as one approached $\gamma_{j}$ so that the $\widetilde{g}_{j}$ have the appropriate degeneracy, cf. [39, Thm.3].

In the context of the conductivity or Helmholtz equations, we can then compare solutions $u$ on $\mathcal{M}$ and $\widetilde{u}$ on $\mathcal{N}$, while for Maxwell's equations we can compare fields $(E, H)$ on $\mathcal{M}$ (with $\varepsilon$ and $\mu$ corresponding to $g$ by the formula of form $(35))$ and $(\widetilde{E}, \widetilde{H})$ on $\mathcal{N}$. For notational convenience, we refer below to the fields as just $u$. 
Metatheorem "A Metatheorem about Metamaterials". If $(\mathcal{M}, \mathcal{N}, \mathcal{F})$ is an STO triplet, there is a 1-1 correspondence, given by $u=\widetilde{u} \circ \mathcal{F}$, i.e., $\left.u\right|_{M_{j}}=\left(\left.\widetilde{u}\right|_{N_{j}}\right) \circ F_{j}$, between finite energy solutions $\widetilde{u}$ to the equation(s) on $\mathcal{N}$, with source terms $\widetilde{f}$ supported on $\mathcal{N} \backslash \Sigma$, and finite energy solutions $u$ on $\mathcal{M}$, with source terms $f=\tilde{f} \circ \mathcal{F}$, satisfying certain "hidden" boundary conditions on $\partial M=\cup_{j=1}^{k} \partial M_{j}$.

\section{$7 \quad$ Further developments}

The literature on metamaterials, cloaking and transformation optics has grown enormously in the last few years. We briefly describe here only some of the highlights.

(a) Although the first descriptions of the cloaking phenomenon were in the context of electrostatics, no proposals of electrostatic metamaterials that might be physically implement the examples of $[38,39]$ have been made to date. [106] does contain a proposal for metamaterials suitable for magnetostatics (cloaking for which is of course mathematically identical to electrostatics) and magnetism at very low nonzero frequencies.

(b) There have been a number of papers in the physics literature theoretically analyzing spherical and cylindrical cloaking. As noted above, [87], which preceded [34], also considered approximate cylindrical cloaking, using it to verify the ideal cloak for a passive object but also exhibiting the instability when no boundary condition is imposed. A scattering theory derivation of the surface currents that arise in cylindrical cloaking was given in [109]. On the other hand, [108] described the scattering characteristics of the simplified "reduced cylindrical parameters", which the experiment [88] was designed to implement, and showed that in fact cloaking with the reduced parameters (which do not arise from transformation optics, but were proposed to replicate the ray behavior of the ideal cloak while using material parameters easier to physically realize) fails even for passive objects. Spherical cloaking of a passive object was analyzed in terms of Mie scattering in [23], and cloaking of a specific active object (an electric dipole) in [110], which rederived (43). A somewhat different treatment of some of these same issues is in [107].

(c) Due to the non-existence of finite energy distributional solutions for 
generic internal currents $\widetilde{J}$, analyzing approximate cloaking in the $3 \mathrm{D}$ spherical geometry would be important, to see if some of the fields $E, H, D$ or $B$ blow up in the limit, as happens in the cylindrical case, see Fig. 4. The blow up would indicate that the linings, e.g., adding very conductive materials at the cloaking surface $\Sigma$, would be needed to regulate the behavior of the fields to help a physical device function more effectively, possibly also improving the function by reducing the far field of the scattered waves, as happens in the cylindrical case.

(d) Other boundary conditions at the cloaking surface, analyzed in the time domain, based on Von Neumann's theory of self-adjoint extensions and using a different notion of solution than that considered here, have been studied in [102, 103, 104]. See also [107].

(e) We have considered singular transformations with range $N_{1}$, where the boundary measurements are being made at the outer boundary of $N_{1}$. In situations where the measurements are made further from cloaked object, [18] introduced, for spherical cloaking, transformations nonlinear in the radial variable in order to give better impedance matching with the surrounding media, and this was further explored for cylindrical cloaking in [108].

(f) Two of the most important practical limitations on cloaking are the narrow bandwidth and lossy nature of currently available metamaterials. Some theoretical analysis of the former issue is in [21].

(g) There has been a drive to design and fabricate metamaterials which function at higher frequencies, with the visible optical range a goal for obvious reasons. Metamaterials with suitable permeability $\mu$ are a particular challenge [90]. [17] gives a proposal for a non-magnetic cloak at optical frequencies; an experiment [93] based on a variant of this design has been reported. More progress on metamaterials in the optical or near-optical range has been obtained in [41] and [68,91].

(h) Cloaking using media with negative index of refraction has been proposed in [77]. Metamaterials and cloaking constructions have also been proposed for other wave phenomena, such as acoustics. See [71, 27, 70], as well as footnote $(1)$ in $\S 1$.

(i) Negative index of refraction material (NIM) has also received a great deal of publicity due to its role in the perfect lens, an idea introduced by 
Pendry [82], building on the earlier work of Veselago [105] where NIMs were first discussed. The perfect lens is a proposal for beating the diffraction resolution limit of one-half the wavelength, using a lens consisting of a flat slab of NIM. That such superresolution might be possible had been suggested earlier $[16,75,76,73]$, but the NIM proposal has been the focus of much theoretical and experimental activity, see also $[50,86]$. Although not without continuing controversy [25], it is now generally accepted to be both theoretically valid and experimentally verified, even for visible light [31].

(j) Effective medium theory for metamaterials is in its early development, and seems to be particularly difficult for materials assembled from periodic or almost-periodic arrays of small cells whose properties are based on resonance effects. A physical (although mathematically nonrigorous) analysis of this kind of media is in [92], which makes implicit assumptions about the smoothness of the fields which are violated when the fields experience the blow up demonstrated in $[87,34]$. Some recent work on homogenization in this context is in [56].

(k) A number of papers have emphasized the use of singular transformation optics designs beyond cloaking. Besides [66], see [84, 85, 51] for designs in two dimensions.

(1) In $\S 6$ we considered transformation optics when the material parameters are blown up on submanifolds. Naturally, rigorous versions of the Metatheorem, with the correct hidden boundary conditions determined, can only be obtained once the details of the designs have been specified. New singular transformation optics devices, with effects on wave propagation previously unknown, lie waiting to be invented!

\section{References}

[1] A. Alu and N. Engheta, Achieving transparency with plasmonic and metamaterial coatings, Phys. Rev. E, 72, 016623 (2005)

[2] K. Astala and L. Päivärinta: Calderón's inverse conductivity problem in the plane. Annals of Math., 163 (2006), 265-299. 
[3] K. Astala, M. Lassas and L. Päivärinta, Calderón's inverse problem for anisotropic conductivity in the plane, Comm. Partial Differential Equations 30 (2005), 207-224.

[4] K. Astala, M. Lassas and L. Päivärinta, Limits of visibility and invisibility for Calderón's inverse problem in the plane, in preparation.

[5] M. Belishev and Y. Kurylev, To the reconstruction of a Riemannian manifold via its spectral data (B-method), Comm. Partial Differential Equations, 17 (1992), 767-804.

[6] Y. Berezanskii, The uniqueness theorem in the inverse problem of spectral analysis for the Schrödinger equation. (Russian) Trudy Moskov. Mat. Obsch., 7 (1958), 1-62.

[7] Y. Benveniste and T. Miloh, Neutral inhomogeneities in conduction phenomeno, Jour. Mech. Phys. Solids., 47 (1999), 1873.

[8] L. Borcea, Electrical impedance tomography, Inverse Problems, 18 (2002), R99-R136.

[9] G. Bouchitte, D. Felbacq, Homogenization of a set of parallel fibers, Waves in Random Media, 7, 2 (1997), 1-12.

[10] G. Bouchitte, D. Felbacq, Homogenization near resonances and artificial magnetism from dielectrics. C. R. Math. Acad. Sci. Paris 339 (2004), $377-382$.

[11] G. Bouchitte, D. Felbacq, Left handed media and homogenization of photonic crystals, Optics letters 30 (2005), 1189-1191.

[12] G. Bouchitte, D. Felbacq, Homogenization of wire mesh photonic crystals embdedded in a medium with a negative permeability, Phys. Rev. Lett. 94 (2005), 183902.

[13] G. Bouchitte, D. Felbacq, Negative refraction in periodic and random photonic crystals, New J. Phys. 7 (2005) 159, 10.1088.

[14] R. Brown and R. Torres, Uniqueness in the inverse aiconductivity problem for conductivities with $3 / 2$ derivatives in $L^{p}, p>2 n$, J. Fourier Analysis Appl., 9(2003), 1049-1056. 
[15] R. Brown and G. Uhlmann, Uniqueness in the inverse conductivity problem with less regular conductivities in two dimensions, Comm. Partial Differential Equations, 22(1997), 1009-10027.

[16] O. Bruno and S. Lintner, Superlens-cloaking of small dielectric bodies in the quasistatic regime. Journal of Applied Physics, 102 (2007), (12). Art. No. 124502.

[17] W. Cai, U. Chettiar, A. Kildishev and V. Shalaev, Optical cloaking with metamaterials, Nature Photonics, 1 (2007), 224-227.

[18] W. C, U. Chettiar, A. Kildishev, G. Milton and V. Shalaev, Non-magnetic cloak without reflection, http://arxiv.org/abs/0707.3641 (2007).

[19] A.P. Calderón, On an inverse boundary value problem, Seminar on Numerical Analysis and its Applications to Continuum Physics (Rio de Janeiro, 1980), pp. 65-73, Soc. Brasil. Mat., Río de Janeiro, 1980.

[20] H. Chen and C.T. Chan, Transformation media that rotate electromagnetic fields, Appl. Phys. Lett. 90 (2007), 241105.

[21] H. Chen, Z. Liang, P. Yao, X. Jiang, H. Ma and C.T. Chan, Extending the bandwidth of electromagnetic cloaks, Phys. Rev. B, 76 (2007), 241104(R).

[22] H. Chen and C.T. Chan, Acoustic cloaking in three dimensions using acoustic metamaterials, Appl. Phys. Lett. 91 (2007), 183518.

[23] H.-S. Chen, B.-I. Wu, B. Zhang and J.A. Kong, Electromagnetic wave interactions with a metamaterial cloak, Phys. Rev. Lett., 99 (2007), 063903.

[24] M. Cheney, D. Isaacson and J. Newell, Electrical impedance tomography, SIAM Rev. 41 (1999), 85-101.

[25] A. Cho, Hope dims for the perfect lens, ScienceNow, http://sciencenow.sciencemag.org/cgi/content/full/2007/423/2.

[26] S. Cummer, B.-I. Popa, D. Schurig, D. Smith and J. Pendry, Fullwave simulations of electromagnetic cloaking structures, Phys. Rev. E 74, 036621 (2006). 
[27] S. Cummer and D. Schurig, One path to acoustic cloaking, New Jour. Phys., 9 (2007), 45.

[28] S. Cummer, et al., Scattering theory derivation of a 3D acoustic cloaking shell, Phys. Rev. Lett., 100 (2008), 024301.

[29] A. Einstein, N. Rosen, The Particle Problem in the General Theory of Relativity. Physical Review 48 (1935), 73.

[30] G. Eleftheriades and K. Balmain, eds., Negative-Refraction Metamaterials, IEEE/Wiley, Hoboken (2005).

[31] N. Fang, H. Lee, C. Sun, X. Zhang, Subdiffraction-limited optical imaging with a silver superlens, Science 308 (2005), 534-537.

[32] A. Greenleaf, Y. Kurylev, M. Lassas and G. Uhlmann, Full-wave invisibility of active devices at all frequencies. Comm. Math. Phys. 275 (2007), 749-789.

[33] A. Greenleaf, Y. Kurylev, M. Lassas and G. Uhlmann, Electromagnetic wormholes and virtual magnetic monopoles from metamaterials. Phys. Rev. Lett. 99, 183901 (2007).

[34] A. Greenleaf, Y. Kurylev, M. Lassas and G. Uhlmann, Improvement of cylindrical cloaking with the SHS lining. Optics Express 15 (2007), 12717-12734.

[35] A. Greenleaf, Y. Kurylev, M. Lassas and G. Uhlmann, Electromagnetic wormholes via handlebody constructions, Comm. Math. Phys. 281 (2008), 369-385

[36] A. Greenleaf, Y. Kurylev, M. Lassas and G. Uhlmann, Comment on "Scattering theory derivation of a 3D acoustic cloaking shell", http://arXiv.org/abs/0801.3279 (2008).

[37] A. Greenleaf, M. Lassas and G. Uhlmann, The Calderón problem for conormal potentials, I: Global uniqueness and reconstruction, Comm. Pure Appl. Math 56 (2003), no. 3, 328-352.

[38] A. Greenleaf, M. Lassas and G. Uhlmann, Anisotropic conductivities that cannot detected in EIT, Physiolog. Meas. (special issue on Impedance Tomography), 24 (2003), 413-420. 
[39] A. Greenleaf, M. Lassas and G. Uhlmann, On nonuniqueness for Calderón's inverse problem, Math. Res. Lett. 10 (2003), no. 5-6, 685693.

[40] I. Hänninen, I. Lindell and A. Sihvola, Realization of Generalized Softand-Hard Boundary, Prog. Electromag. Res., PIER 64, 317 (2006).

[41] A. Hoffman, et al., Negative refraction in semiconductor metamaterials, Nature Materials, doi:10.1038/nmat2033 (14 Oct 2007).

[42] D. Isaacson, J. Mueller and S. Siltanen, Special issue on electrical impedance tomography of Physiological Measurement, 24, 2003

[43] A. Jenkins, Lost in Space, Nature Photonics, 2, 11-11 (01 Jan 2008).

[44] A. Kachalov and Y. Kurylev, Multidimensional inverse problem with incomplete boundary spectral data, Comm. Part. Diff. Eq., 23 (1998), $55-95$.

[45] A. Kachalov, Y. Kurylev and M. Lassas, Inverse Boundary Spectral Problems, Chapman and Hall/CRC Monogr. and Surv. in Pure and Appl. Math., 123. Chapman and Hall/CRC, Boca Raton, 2001. xx+290 pp.

[46] M. Kerker, Invisible bodies, J. Opt. Soc. Am., 65 (1975), 376.

[47] P.-S. Kildal, Definition of artificially soft and hard surfaces for electromagnetic waves, Electron. Lett. 24 (1988), 168-170.

[48] P.-S. Kildal, Artificially soft and hard surfaces in electromagnetics, IEEE Trans. Ant. and Prop., 38 (1990), no. 10, 1537-1544.

[49] P.-S. Kildal, A. Kishk, Z. Sipus, RF invisibility using metamaterials: Harry Potter's cloak or the Emperor's new clothes?, IEEE APS Int. Symp., Hawaii, June, 2007.

[50] A.V. Kildidhev and E.E. Narimanov, Impedance-matched hyperlens, http://arxiv.org/abs/0708.3798

[51] A. Kildishev and V. Shalaev, Engineering space for light via transformation optics, Opt. Lett., 33 (2007), 43-45. 
[52] T. Kilpeläinen, J. Kinnunen and O. Martio, Sobolev spaces with zero boundary values on metric spaces. Potential Anal. 12 (2000), 233-247.

[53] V, Kolehmainen, M. Lassas and P. Ola, Inverse conductivity problem with an imperfectly known boundary, SIAM J. Appl. Math., 66 (2005), $365-383$.

[54] R. Kohn, D. Onofrei, M. Vogelius and M. Weinstein, Cloaking via change of variables for the Helmholtz equation, in preparation.

[55] R. Kohn, H. Shen, M. Vogelius and M. Weinstein, Cloaking via change of variables in electrical impedance tomography, Inver. Prob., 24 (2008), 015016.

[56] R. Kohn and S. Shipman, Magnetism and homogenization of microresonators, http://arXiv.org/abs/0712.2210v1 (2007).

[57] R. Kohn, M. Vogelius, Determining conductivity by boundary measurements II, Interior results, Comm. Pure Appl. Math., 38 (1985), 643-667.

[58] R. Kohn, M. Vogelius, Identification of an unknown conductivity by means of measurements at the boundary, in Inverse Problems, SIAMAMS Proc., 14 (1984).

[59] Y. Kurylev, Multidimensional inverse boundary problems by the BCmethod: groups of transformations and uniqueness results, Math. Comput. Modelling, 18 (1993), 33-46.

[60] Y. Kurylev, M. Lassas and E. Somersalo, Maxwell's equations with a polarization independent wave velocity: Direct and inverse problems, Jour. de Math. Pures et Appl., 86 (2006), 237-270.

[61] M. Lassas and G. Uhlmann, Determining Riemannian manifold from boundary measurements, Ann. Sci. École Norm. Sup., 34 (2001), 771787.

[62] M. Lassas, M. Taylor and G. Uhlmann, The Dirichlet-to-Neumann map for complete Riemannian manifolds with boundary, Comm. Geom. Anal., 11 (2003), 207-222. 
[63] R. Lavine and A. Nachman, The Faddeev-Lipmann-Schwinger equation in multidimensional quantum inverse scattering, in Inverse problems: an interdisciplinary study (Montpellier, 1986), 169-174.

[64] J. Lee and G. Uhlmann, Determining anisotropic real-analytic conductivities by boundary measurements, Comm. Pure Appl. Math., 42 (1989), 1097-1112.

[65] U. Leonhardt, Optical Conformal Mapping, Science 312 (23 June, 2006), 1777-1780.

[66] U. Leonhardt and T. Philbin, General relativity in electrical engineering, New J. Phys., 8 (2006), 247; doi:10.1088/1367-2630/8/10/247.

[67] I. Lindell, Generalized soft-and-hard surface, IEEE Tran. Ant. and Propag., 50 (2002), 926-929.

[68] N. Liu, H. Guo, L. Fu, S. Kaiser, H. Schweizer and H. Giessen, Threedimensional photonic metamaterials at optical frequencies, Nature $\mathrm{Ma}$ terials, 7 (2008), 31-37.

[69] D. Miller, On perfect cloaking, Opt. Exp., 14 (2006),12457-12466.

[70] G. Milton, New metamaterials with macroscopic behavior outside that of continuum elastodynamics, New Jour. Phys., 9 (2007), 359.

[71] G. Milton, M. Briane and J. Willis, On cloaking for elasticity and physical equations with a transformation invariant form, New J. Phys., 8 (2006), 248; doi:10.1088/1367-2630/8/10/248.

[72] G. Milton and N.-A. Nicorovici, On the cloaking effects associated with anomalous localized resonance, Proc. Royal Soc. A 462 (2006), 30273059 .

[73] G. Milton, N.-A. Nicorovici and R. McPhedran, Opaque perfect lenses, to appear in Physica B, arXiv:physics/0608225v2.

[74] A. Nachman, Global uniqueness for a two-dimensional inverse boundary value problem, Ann. of Math. 143 (1996), 71-96. 
[75] N. Nicorovici, R. McPhedran and G. Milton, Optical and dielectric properties of partially resonant composites. Phys. Rev. B 49 (1994), 84798482 .

[76] N. Nicorovici, G. Milton, R. McPhedran and L. Botten, Quasistatic cloaking of ¿two-dimensional polarizable discrete systems by anomalous resonance, Opt. Express 15 (2007), 6314-6323.

[77] T. Ochiai, U. Leonhardt and J. Nacher, A novel design of dielectric perfect invisibility devices, http://arxiv.org/abs/0711.1122 (2007).

[78] P. Ola, L. Päivärinta and E. Somersalo, An inverse boundary value problem in electrodynamics. Duke Math. J. 70 (1993), no. 3, 617-653.

[79] L. Päivärinta, A. Panchenko and G. Uhlmann, Complex geometrical optics for Lipschitz conductivities, Rev. Mat. Iberoam., 19 (2003), 57-72.

[80] J.B. Pendry, D. Schurig and D.R. Smith, Controlling electromagnetic fields, Science 312 (23 June, 2006), 1780 - 1782.

[81] J.B. Pendry, D. Schurig and D.R. Smith, Calculation of material properties and ray tracing in transformation media, Opt. Exp. 14, 9794 (2006).

[82] J. Pendry, Negative refraction makes a perfect lens, Phys. Rev. Lett. 8 (2000), 3966 - 3969.

[83] Physorg.com, The Mathematics of Cloaking, http:// www.physorg.com/news86358402.html (Dec. 26, 2006).

[84] M. Rahm, D. Schurig, D. Roberts, S. Cummer, D. Smith, J. Pendry, Design of electromagnetic cloaks and concentrators using form-invariant coordinate transformations of Maxwell's equations, Photonics and Nanostructures, 6 /2008), 87-95.

[85] M. Rahm, S. Cummer, D. Schurig, J. Pendry and D. Smith, Optical design of reflectionless complex media by finite embedded coordinate transformations, preprint, Phys. Rev. Lett., 100 (2008), 063903.

[86] M. Rahm, D. Schurig, D.A. Roberts, S.A. Cummer, D.R. Smith and J. Pendry, Design of Electromagnetic Cloaks and Concentrators Using Form-Invariant Coordinate Transformations of Maxwell's Equations, arxiv:0706.2452v1. 
[87] Z. Ruan, M. Yan, C. Neff and M. Qiu, Ideal cylindrical cloak: perfect but sensitive to tiny perturbations, Phys. Rev. Lett. 99 (2007), 113903.

[88] D. Schurig, J. Mock, B. Justice, S. Cummer, J. Pendry, A. Starr and D. Smith, Metamaterial electromagnetic cloak at microwave frequencies, Science $\mathbf{3 1 4}$ (2006), no. 5801, pp. 977-980.

[89] D. Schurig, J. Pendry, D.R. Smith, Transformation-designed optical elements Optics Express, 15 (2007), Issue 22, 14772-14782.

[90] V. Shalaev, W. Cai, U. Chettiar, H.-K. Yuan, A. Sarychev, V. Drachev and A. Kildishev, Negative index of refraction in optical metamaterials Opt. Lett., 30 (2005), 3356-3358

[91] G. Shvets, Metamaterials add an extra dimension, Nature Materials, 7 (Jan. 2008), 7-8.

[92] D. Smith and J. Pendry, Homogenization of metamaterials by field averaging, Jour. Opt. Soc. Am. B, 23 (2006), 391-403.

[93] I. Smolyaninov, Y. Hung and C. Davis, Electromagnetic cloaking in the visible frequency range, http://arxiv.org/abs/0709.2862 (2007).

[94] Z. Sun and G. Uhlmann, Anisotropic inverse problems in two dimensions, Inverse Problems, 19 (2003), 1001-1010.

[95] J. Sylvester, An anisotropic inverse boundary value problem, Comm. Pure Appl. Math. 43 (1990), 201-232.

[96] J. Sylvester and G. Uhlmann, A global uniqueness theorem for an inverse boundary value problem. Ann. of Math. 125 (1987), 153-169.

[97] K. Tsakmakidis and O. Hess, Optics: Watch your back, Nature 451, 27 (3 January 2008), doi:10.1038/451027a.

[98] G. Uhlmann, Scattering by a metric, Chap. 6.1.5, in Encyclopedia on Scattering, R. Pike and P. Sabatier, eds., Academic Pr. (2002), 16681677.

[99] G. Uhlmann, Developments in inverse problems since Calderón's foundational paper, Chapter 19 in Harmonic Analysis and Partial Differential Equations, M. Christ, C. Kenig and C. Sadosky, eds., University of Chicago Press (1999), 295-345 . 
[100] R. Walser, in: W.S. Weiglhofer and A. Lakhtakia (Eds.), Introduction to Complex Mediums for Electromagnetics and Optics, SPIE Press, Bellingham, WA, USA, 2003

[101] A. Ward and J. Pendry, Refraction and geometry in Maxwell's equations, Jour. Modern Opt., 43 (1996), 773-793.

[102] R. Weder, A rigorous time-domain analysis of full-wave electromagnetic cloaking (Invisibility), http://arXiv.org/abs/0704.0248v1,2,3,4.

[103] R. Weder, A rigorous analysis of high order electromagnetic invisibility cloaks, Jour. Phys. A: Math. Theor., 41 (2008), 065207.

[104] R. Weder, The boundary conditions for electromagnetic invisibility cloaks, http://arxiv.org/abs/0801.3611 (2008).

[105] V.G. Veselago, Sov. Phys. Usp. 10 (1968), 509.

[106] B. Wood and J. Pendry, Metamaterials at zero frequency, Jour. Phys.:Condens Matter, 19 (2007), 076208.

[107] A. Yaghjian and S. Maci, Alternative derivation of electromagnetic cloaks and concentrators, http://arxiv.org/abs/0710.2933v1,2 (2007).

[108] M. Yan, Z. Ruan and M. Qiu, Scattering characteristics of simplified cylindrical invisibility cloaks, Opt. Exp., 15 (2007), 17772.

[109] B. Zhang, et al., Response of a cylindrical invisibility cloak to electromagnetic waves, Phys. Rev. B, 76 (2007), 121101(R).

[110] B. Zhang, et al., Extraordinary surface voltage effect in the invisibility cloak with an active device inside, Phys. Rev. Lett., 100 (2008), 063904.

[111] F. Zolla, S, Guenneau, A. Nicolet and J. Pendry, Electromagnetic analysis of cylindrical invisibility cloaks and the mirage effect, Opt. Lett., 32 (2007), 1069-1071. 